\title{
Local Noncollinear Spin Analysis
}

\author{
Bayileyegn A. Abate, ${ }^{\dagger,}$ Rajendra P. Joshi, ${ }^{\ddagger}$ and Juan E. Peralta*, \\ $\dagger$ Science of Advanced Materials, Central Michigan University, Mount Pleasant, MI, 48859, \\ $U S A$ \\ $\ddagger$ Department of Physics, Central Michigan University, Mount Pleasant, MI, 48859, USA \\ IBoth authors contributed equally to this work. \\ E-mail: peral1j@cmich.edu
}

\begin{abstract}
In this work, we generalize the local spin analysis of Clark and Davidson [J. Chem. Phys. 115(16), 7382 (2001)] for the partitioning of the expectation value of the molecular spin square operator, $\left\langle\hat{\mathbf{S}}^{2}\right\rangle$, into atomic contributions, $\left\langle\hat{\mathbf{S}}_{A} \cdot \hat{\mathbf{S}}_{B}\right\rangle$, to the noncollinear spin case in the framework of density functional theory (DFT). We derive the working equations and we show applications to the analysis of the noncollinear spin solutions of typical spin-frustrated systems and to the calculation of magnetic exchange couplings. In the former case, we employ the triangular $\mathrm{H}_{3} \mathrm{He}_{3}$ test molecule and a $\mathrm{Mn}_{3}$ complex to show that the local spin analysis provides additional information that complements the standard one-particle spin population analysis. For the calculation of magnetic exchange couplings, $J_{A B}$, we employ the local spin partitioning to extract $\left\langle\hat{\mathbf{S}}_{A} \cdot \hat{\mathbf{S}}_{B}\right\rangle$ as a function of the interatomic spin orientation given by the angle $\theta$. This, combined with the dependence of the electronic energy with $\theta$, provides a methodology to extract $J_{A B}$ from DFT calculations that, in contrast to conventional energy differences based methods, does not require the use of ad-hoc $S_{A}$ and $S_{B}$ values.
\end{abstract}




\section{Introduction}

Molecular magnets, spin-glasses, and topologically frustrated anti-ferromagnets are representative examples of materials exhibiting noncollinear magnetism, where the spins may be disordered and the direction of the magnetization density varies in space. ${ }^{14}$ Noncollinearity in the spin direction usually originates in the geometric frustration of anti-ferromagnetic interactions, magnetic anisotropy effects, or are induced for particular device applications. ${ }^{5}$ Although electronic structure methods needed to deal with such systems naturally involve the use of multi-determinant wave functions, the typical size and complexity of these systems prohibit the use of multi-reference wave function methods, and practical calculations are limited to single-determinant methods. $\frac{617}{6 v e r}$ the years, density-functional theory (DFT) has become one of the most successful and widely used computational tools for electronic structure theory of complex chemical systems, mainly due to the combination of its low computational cost and the availability of increasingly accurate approximations to the exchange-correlation energy. ${ }^{[11]}$ Within the DFT formalism, a general description of the spin degree of freedom can be realized by allowing noncollinear spin magnetization. $\stackrel{21216}{12}$ This generalization, refered to as noncollinear spin DFT, helped to gain insight into the underlying physics of materials properties and chemical processes involving magnetic systems. 212 Although a wealth of DFT calculations currently employ the noncollinear spin formalism, the analysis of the resulting spin density is limited to the partitioning of the expectation value of the one-particle spin operator, $\langle\hat{\mathbf{S}}\rangle$. This analysis provides information about the magnitude and direction of the spin magnetization of different atoms or molecular units but lacks information about interatomic spin interactions.

The concept of local spins $\sqrt{6 \mid 19} \sqrt[23]{23}$ is based on the partitioning of the expectation value

of the molecular spin square operator, $\left\langle\hat{\mathbf{S}}^{2}\right\rangle$, and provides a valuable input to understand the electronic structure of molecules that complements the information obtained from oneparticle population analysis methods.

In Kohn-Sham (KS) DFT, $\left\langle\hat{\mathbf{S}}^{2}\right\rangle$ is calculated in analogy to ab initio wave function meth- 
ods utilizing the auxiliary KS orbitals. ${ }^{24}$ It should be pointed out that, although this is common practice, in DFT the formally correct way to evaluate $\left\langle\hat{\mathbf{S}}^{2}\right\rangle$ is trhough a functional of the spin-density. $\frac{25}{27}$ Local spin analyses rely on the exact decomposition of the $\hat{\mathbf{S}}$ operator in terms of local projectors. $\frac{19}{2128} 34$ The general framework originally proposed by Clark and Davidson ${ }^{19}$ employs Hermitian one-electron projection operators to decompose $\left\langle\hat{\mathbf{S}}^{2}\right\rangle$ into one- and two-center contributions, $\left\langle\hat{\mathbf{S}}_{A}^{2}\right\rangle$ and $\left\langle\hat{\mathbf{S}}_{A} \cdot \hat{\mathbf{S}}_{B}\right\rangle_{A \neq B}$, respectively. The pioneer method of Clark and Davidson has been used in several contexts to characterize local collinear spins

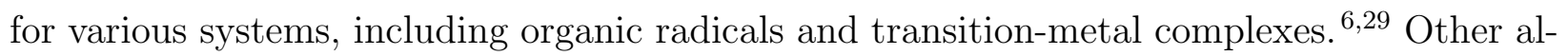
ternative decomposition schemes have been proposed in the literature. ${ }^{21133}$ In this work, we extend the local spin analysis of Clark and Davidson to the general noncollinear spin case. As proof-of-concept, we apply the local spin analysis to the triangular $\mathrm{H}_{3} \mathrm{He}_{3}$ test molecule and a $\mathrm{Mn}_{3}$ complex, both showing noncollinear spin solutions originated by geometrically frustrated anti-ferromagnetic interactions. We also show that the local noncollinear spin analysis can be used as a tool to extract magnetic exchange coupling parameters from a unique single-reference high spin state without ad-hoc assumptions about nominal $S_{A}$ and $S_{B}$ spin values.

\section{Theory}

In noncollinear spin DFT, spin noncollinearity is introduced through two-component KohnSham complex spinors

$$
\psi_{i}(\mathbf{r})=\left(\begin{array}{c}
\psi_{i}^{\uparrow}(\mathbf{r}) \\
\psi_{i}^{\downarrow}(\mathbf{r})
\end{array}\right)
$$

where the spatial orbitals, $\psi_{i}^{\uparrow}(\mathbf{r})$ and $\psi_{i}^{\downarrow}(\mathbf{r})$ are expanded in terms of atomic orbitals,

$$
\psi_{i}^{\sigma}(\mathbf{r})=\sum_{\nu} c_{\nu i}^{\sigma} \phi_{\nu}(\mathbf{r}) \quad(\sigma=\uparrow, \downarrow)
$$


For the purpose of this work, it is convenient to use the one-electron density matrix

$$
D_{\mu \nu}=\sum_{i \in o c c}\left(\begin{array}{cc}
c_{\mu i}^{\uparrow} c_{\nu i}^{\uparrow *} & c_{\mu i}^{\uparrow} c_{\nu i}^{\downarrow *} \\
c_{\mu i}^{\downarrow} c_{\nu i}^{\uparrow *} & c_{\mu i}^{\downarrow} c_{\nu i}^{\downarrow *}
\end{array}\right)=\left(\begin{array}{cc}
\mathbf{D}_{\mu \nu}^{\uparrow \uparrow} & \mathbf{D}_{\mu \nu}^{\uparrow \downarrow} \\
\mathbf{D}_{\mu \nu}^{\downarrow \uparrow} & \mathbf{D}_{\mu \nu}^{\downarrow \downarrow}
\end{array}\right),
$$

where $\mathbf{D}_{\mu \nu}^{\sigma \sigma^{\prime}}$ are the four spin blocks of the complex density matrix used in this local noncollinear spin partitioning.

The local projection operator associated with atom $A, \hat{P}_{A}$ is used to project the contribution of atom $A$ from the total molecular spin. The total and local spin operators can be written as

$$
\begin{aligned}
\hat{\mathbf{S}} & =\sum_{i} \hat{\mathbf{S}}(i), \text { and } \\
\hat{\mathbf{S}}_{A} & =\sum_{i} \hat{\mathbf{S}}(i) \hat{P}_{A}(i) .
\end{aligned}
$$

Using this definition of $\hat{\mathbf{S}}_{A}$, the square of the total spin operator becomes

$$
\hat{\mathbf{S}}^{2}=\sum_{A, B} \hat{\mathbf{S}}_{A} \cdot \hat{\mathbf{S}}_{B}
$$

which can be expanded in terms of local projection operators using

$$
\begin{aligned}
\hat{\mathbf{S}}_{A} \cdot \hat{\mathbf{S}}_{B} & =\sum_{i j} \hat{\mathbf{S}}(i) \hat{P}_{A}(i) \cdot \hat{\mathbf{S}}(j) \hat{P}_{B}(j) \\
& =\delta_{A B} \sum_{i} \hat{P}_{A}(i) \hat{S}^{2}(i)+\frac{1}{2} \sum_{i, j} \hat{\mathbf{S}}(i) \cdot \hat{\mathbf{S}}(j)\left[\hat{P}_{A}(i) \hat{P}_{B}(j)+\hat{P}_{B}(i) \hat{P}_{A}(j)\right]
\end{aligned}
$$

In Eq. (7), the first and second terms on the right-hand side represent one- and two-electron operators, respectively. For a single-reference method, such as Hartree-Fock or Kohn-Sham 
$\mathrm{DFT}, \stackrel{910}{,}$ the expectation value $\left\langle\hat{\mathbf{S}}_{A} \cdot \hat{\mathbf{S}}_{B}\right\rangle$ is given by

$$
\begin{aligned}
\left\langle\hat{\mathbf{S}}_{A} \cdot \hat{\mathbf{S}}_{B}\right\rangle= & \frac{3}{4} \delta_{A B} \sum_{m}\left\langle m\left|\hat{P}_{A}\right| m\right\rangle+\frac{1}{2} \sum_{m, n}\left\langle m n\left|\hat{\mathbf{S}}(1) \cdot \hat{\mathbf{S}}(2)\left[\hat{P}_{A} \hat{P}_{B}+\hat{P}_{B} \hat{P}_{A}\right]\right| m n\right\rangle \\
& -\frac{1}{2} \sum_{m, n}\left\langle m n\left|\hat{\mathbf{S}}(1) \cdot \hat{\mathbf{S}}(2)\left[\hat{P}_{A} \hat{P}_{B}+\hat{P}_{B} \hat{P}_{A}\right]\right| n m\right\rangle,
\end{aligned}
$$

where $m$ and $n$ refer to two-component spinors, Eq. (1). Using the fact that spin and projection operators commute, and working the algebra for the two-component spinors, the expectation value $\left\langle\hat{\mathbf{S}}_{A} \cdot \hat{\mathbf{S}}_{B}\right\rangle$ in terms of the projection operators, $\hat{P}_{A}$ and $\hat{P}_{B}$, can be cast as

$$
\begin{aligned}
\left\langle\hat{\mathbf{S}}_{A} \cdot \hat{\mathbf{S}}_{B}\right\rangle= & \frac{3}{4} \delta_{A B} \sum_{m}\left[\left\langle m^{\uparrow}\left|\hat{P}_{A}\right| m^{\uparrow}\right\rangle+\left\langle m^{\downarrow}\left|\hat{P}_{A}\right| m^{\downarrow}\right\rangle\right]+ \\
& \sum_{m, n}\left[\frac{1}{4}\left\langle m^{\uparrow}\left|\hat{P}_{A}\right| m^{\uparrow}\right\rangle\left\langle n^{\uparrow}\left|\hat{P}_{B}\right| n^{\uparrow}\right\rangle+\frac{1}{4}\left\langle m^{\downarrow}\left|\hat{P}_{A}\right| m^{\downarrow}\right\rangle\left\langle n^{\downarrow}\left|\hat{P}_{B}\right| n^{\downarrow}\right\rangle\right. \\
& -\frac{1}{4}\left\langle m^{\uparrow}\left|\hat{P}_{A}\right| n^{\uparrow}\right\rangle\left\langle n^{\uparrow}\left|\hat{P}_{B}\right| m^{\uparrow}\right\rangle-\frac{1}{4}\left\langle m^{\downarrow}\left|\hat{P}_{A}\right| n^{\downarrow}\right\rangle\left\langle n^{\downarrow}\left|\hat{P}_{B}\right| m^{\downarrow}\right\rangle \\
& -\frac{1}{4}\left\langle m^{\uparrow}\left|\hat{P}_{A}\right| m^{\uparrow}\right\rangle\left\langle n^{\downarrow}\left|\hat{P}_{B}\right| n^{\downarrow}\right\rangle-\frac{1}{4}\left\langle m^{\downarrow}\left|\hat{P}_{A}\right| m^{\downarrow}\right\rangle\left\langle n^{\uparrow}\left|\hat{P}_{B}\right| n^{\uparrow}\right\rangle \\
& -\frac{1}{2}\left\langle m^{\uparrow}\left|\hat{P}_{A}\right| n^{\uparrow}\right\rangle\left\langle n^{\downarrow}\left|\hat{P}_{B}\right| m^{\downarrow}\right\rangle-\frac{1}{2}\left\langle m^{\downarrow}\left|\hat{P}_{A}\right| n^{\downarrow}\right\rangle\left\langle n^{\uparrow}\left|\hat{P}_{B}\right| m^{\uparrow}\right\rangle \\
& +\frac{1}{2}\left\langle m^{\uparrow}\left|\hat{P}_{A}\right| m^{\downarrow}\right\rangle\left\langle n^{\downarrow}\left|\hat{P}_{B}\right| n^{\uparrow}\right\rangle+\frac{1}{2}\left\langle m^{\downarrow}\left|\hat{P}_{A}\right| m^{\uparrow}\right\rangle\left\langle n^{\uparrow}\left|\hat{P}_{B}\right| n^{\downarrow}\right\rangle \\
& \left.+\frac{1}{4}\left\langle m^{\uparrow}\left|\hat{P}_{A}\right| n^{\downarrow}\right\rangle\left\langle n^{\downarrow}\left|\hat{P}_{B}\right| m^{\uparrow}\right\rangle+\frac{1}{4}\left\langle m^{\downarrow}\left|\hat{P}_{A}\right| n^{\uparrow}\right\rangle\left\langle n^{\uparrow}\left|\hat{P}_{B}\right| m^{\downarrow}\right\rangle\right] .
\end{aligned}
$$

In Eq. 9. $\left|m^{\sigma}\right\rangle$ represents the $\sigma$ space orbital associated with the spinor $\left|\psi_{m}\right\rangle$, so that $\left\langle m^{\sigma}\left|\hat{P}_{X}\right| n^{\sigma^{\prime}}\right\rangle=\int d^{3} r \psi_{m}^{\sigma *}(\mathbf{r}) \hat{P}_{X} \psi_{n}^{\sigma^{\prime}}(\mathbf{r})$. For the purposes of this decomposition procedure, there are certain conditions that the local projection operators are required to fulfill. First, the projection operators should be idempotent and orthogonal. Second, the sum of all projectors must sum up to the identity operator, $\sum_{A} \hat{P}_{A}=\hat{I} .20129$ Using these conditions, the 
final working expression for $\left\langle\hat{\mathbf{S}}_{A} \cdot \hat{\mathbf{S}}_{B}\right\rangle$ is

$$
\begin{aligned}
\left\langle\hat{\mathbf{S}}_{A} \cdot \hat{\mathbf{S}}_{B}\right\rangle= & \frac{3}{4} \delta_{A B} \sum_{\mu \in A}\left[\mathbf{P}_{\mu \mu}^{\uparrow \uparrow}+\mathbf{P}_{\mu \mu}^{\downarrow \downarrow}\right]+\sum_{\mu \in A, \nu \in B}\left[\frac{1}{4} \mathbf{P}_{\mu \mu}^{\uparrow \uparrow} \mathbf{P}_{\nu \nu}^{\uparrow \uparrow}+\frac{1}{4} \mathbf{P}_{\mu \mu}^{\downarrow \downarrow} \mathbf{P}_{\nu \nu}^{\downarrow \downarrow}\right. \\
& -\frac{1}{4} \mathbf{P}_{\mu \nu}^{\uparrow \uparrow} \mathbf{P}_{\nu \mu}^{\uparrow \uparrow}-\frac{1}{4} \mathbf{P}_{\mu \nu}^{\downarrow \downarrow} \mathbf{P}_{\nu \mu}^{\downarrow \downarrow}-\frac{1}{4} \mathbf{P}_{\mu \mu}^{\uparrow \uparrow} \mathbf{P}_{\nu \nu}^{\downarrow \downarrow}-\frac{1}{4} \mathbf{P}_{\mu \mu}^{\downarrow \downarrow} \mathbf{P}_{\nu \nu}^{\uparrow \uparrow}-\frac{1}{2} \mathbf{P}_{\mu \nu}^{\uparrow \uparrow} \mathbf{P}_{\nu \mu}^{\downarrow \downarrow} \\
& \left.-\frac{1}{2} \mathbf{P}_{\mu \nu}^{\downarrow \downarrow} \mathbf{P}_{\nu \mu}^{\uparrow \uparrow}+\frac{1}{2} \mathbf{P}_{\mu \mu}^{\uparrow \downarrow} \mathbf{P}_{\nu \nu}^{\downarrow \uparrow}+\frac{1}{2} \mathbf{P}_{\mu \mu}^{\downarrow \uparrow} \mathbf{P}_{\nu \nu}^{\uparrow \downarrow}+\frac{1}{4} \mathbf{P}_{\mu \nu}^{\uparrow \downarrow} \mathbf{P}_{\nu \mu}^{\downarrow \uparrow}+\frac{1}{4} \mathbf{P}_{\mu \nu}^{\downarrow \uparrow} \mathbf{P}_{\nu \mu}^{\uparrow \downarrow}\right],
\end{aligned}
$$

where $\mathbf{P}_{\eta \zeta}^{\sigma \sigma^{\prime}}$ are the projected one-electron density matrix elements (for a detailed derivation of Eq. 10 please see the Supporting Information) For the collinear spin case (assuming spinpolarization in the $z$ direction), the contributions from the cross-terms in the generalized spin-density matrices are all zero, and hence the last four terms in Eq. 10 vanish, giving an expression that is equivalent to Eq. (16) in the paper of Hermann et al. ${ }^{29}$ For the purpose of implementing the local spin analysis, it is convenient to attempt to compact the notation in Eq. 10. To this end, we define the vector $\overrightarrow{\mathbf{P}}_{\mu \nu}$ of Cartesian components $\mathbf{P}_{\mu \nu}^{x}=\mathbf{P}_{\mu \nu}^{\uparrow \downarrow}+\mathbf{P}_{\mu \nu}^{\downarrow \uparrow}$, $\mathbf{P}_{\mu \nu}^{y}=i\left(\mathbf{P}_{\mu \nu}^{\uparrow \downarrow}-\mathbf{P}_{\mu \nu}^{\downarrow \uparrow}\right), \mathbf{P}_{\mu \nu}^{z}=\mathbf{P}_{\mu \nu}^{\uparrow \uparrow}-\mathbf{P}_{\mu \nu}^{\downarrow \downarrow}$, and the scalar $\mathbf{P}_{\mu \nu}=\mathbf{P}_{\mu \nu}^{\uparrow \uparrow}+\mathbf{P}_{\mu \nu}^{\downarrow \downarrow}$. Using these matrices, Eq. 10 can be reduced to

$$
\left\langle\hat{\mathbf{S}}_{A} \cdot \hat{\mathbf{S}}_{B}\right\rangle=\frac{3}{4}\left(\mathbf{P}_{A A} \delta_{A B}-\frac{1}{2} \mathbf{P}_{A B} \mathbf{P}_{B A}\right)+\frac{1}{4}\left(\overrightarrow{\mathbf{P}}_{A A} \cdot \overrightarrow{\mathbf{P}}_{B B}+\frac{1}{2} \overrightarrow{\mathbf{P}}_{A B} \cdot \overrightarrow{\mathbf{P}}_{B A}\right),
$$

where we have used the fact that the generalized density matrix $\mathbf{P}_{\mu \nu}^{\sigma \sigma}$ is Hermitian, and the indexes $A$ and $B$ imply the summations $\sum_{\mu \in A}$ and $\sum_{\nu \in B}$. It is interesting to note that since $\overrightarrow{\mathbf{P}}_{\mu \nu}$ transforms as a vector, Eq. 11 trivially reflects the rotational invariance of the decomposition of $\left\langle\hat{\mathbf{S}}^{2}\right\rangle$. Eqs. 10 and 11 are general and the main result of this work. Out of several alternatives available in the literature for the choice of the projector, ${ }^{21 / 29133}$ Löwdin projection operators, as originally employed in the work of Clark and Davidson ${ }^{19}$ are among the most widely used and simplest to implement. ${ }^{35}$ Hence, in this work, the implementation has been carried out using Löwdin projectors using $\mathbf{P}^{\sigma \sigma^{\prime}}=\mathbf{S}^{1 / 2} \mathbf{D}^{\sigma \sigma^{\prime}} \mathbf{S}^{1 / 2}$, where $\mathbf{S}$ is the 
overlap matrix.

\section{Computational Details}

The $\left\langle\hat{\mathbf{S}}^{2}\right\rangle$ decomposition scheme presented in the previous section has been implemented in an in-house Gaussian development version. $\stackrel{36}{36}$ Five density functional approximations in combination with the triple-zeta split valence basis set with polarization, $\stackrel{37}{27 Z V P}$, were employed to perform the noncollinear spin calculations on the two benchmark systems: The triangular $\mathrm{H}_{3} \mathrm{He}_{3}$ and the polynuclear oxomanganese complex $\left[\left(\mathrm{Mn}^{4}\right)_{3} \mathrm{O}_{4} \mathrm{~L}_{4}\left(\mathrm{H}_{2} \mathrm{O}\right)\right]\left(\left[\mathrm{Mn}_{3}\right]\right.$ complex). ${ }^{14}$ Both systems can be considered as antiferromagnetically coupled spin trimers with frustrated noncollinear spin configurations. We included in our tests the local spin density approximation (LSDA) built as Slater exchange and the parametrization of Wosko, Wilk, and Nusair ${ }^{38139}$ for correlation, two functionals from the generalized gradient approximation: the Perdew, Burke, and Ernzerhof (PBE) functional $\stackrel{40}{40}$ and the Becke's 1988 exchange plus Perdew's 1996 correlation (BP86),, 4142 and two representative hybrid functionals: PBEh ${ }^{43}$ (PBE hybrid, also refer to as PBE1PBE ${ }^{44}$ and $\mathrm{PBE} 0^{\sqrt[45]{4}}$ in the literature) and B3LYP. $41 \mid 46,49$

In order to find these noncollinear spin configurations in the self-consistent solutions of the KS equations, it is important to start from a suitable initial guess. For this work, the initial guesses were thus generated by pre-conditioning the electron spin density in a noncollinear configuration using a constraint that imposes local-spin moments in pre-selected directions. These constraints are introduced via Lagrange multipliers. To this end, we write the local magnetization for atom $A$ as

$$
\mathbf{m}^{A}=\sum_{\mu, \nu} \mathcal{W}_{\mu \nu}^{A} \mathbf{D}_{\mu \nu}
$$

where $\mathcal{W}^{A}$ is a local projector, $\mathbf{D}_{\mu \nu}$ is the spin-density matrix vector with Cartesian components $D_{\mu \nu}^{x}=D_{\mu \nu}^{\uparrow \downarrow}+D_{\mu \nu}^{\downarrow \uparrow}, D_{\mu \nu}^{y}=i\left(D_{\mu \nu}^{\uparrow \downarrow}-D_{\mu \nu}^{\downarrow \uparrow}\right)$, and $D_{\mu \nu}^{z}=D_{\mu \nu}^{\uparrow \uparrow}-D_{\mu \nu}^{\downarrow \downarrow}$. In Eq. (12), $\mathcal{W}^{A}$ is 
defined from the Löwdin partitioning as

$$
\mathcal{W}_{\mu \nu}^{A}=\sum_{\lambda \in A}\left(\mathbf{S}^{1 / 2}\right)_{\mu \lambda}\left(\mathbf{S}^{1 / 2}\right)_{\lambda \nu}
$$

where $\mathbf{S}$ is the AO overlap matrix. For each atom $A$ for which the local magnetization $\mathbf{m}^{A}$ is to be constrained in a direction $\mathbf{e}^{A}$, an aditional term $\mathbf{h}^{A}$ is included in $\mathbf{H}_{K S}$,

$$
\mathbf{h}^{A}=\mathcal{W}^{A} \boldsymbol{\lambda}^{A} \cdot\left(\boldsymbol{\sigma} \times \mathbf{e}^{A}\right),
$$

where $\mathbf{e}^{A}$ is a unit vector to which $\mathbf{m}^{A}$ is constraint to be parallel to, $\boldsymbol{\lambda}^{A}$ is a Lagrange multiplier vector and $\boldsymbol{\sigma}$ are the Pauli matrices. For simplicity, and for the purpose of generating

an initial guess, in this work we use a fixed value for $\boldsymbol{\lambda}^{A}=0.5$. For the magnetic exchange coupling application in the next Section), two multipliers, $\boldsymbol{\lambda}^{A}$ and $\boldsymbol{\lambda}^{B}$ are introduced (one for each atom $A$ and $B$ ) and the values of $\boldsymbol{\lambda}^{A}$ and $\boldsymbol{\lambda}_{B}$ are variationally optimized to minimize the KS energy during the self-consistent iterations. The total and local spin values reported in this work are in atomic units, and no symmetry was imposed (keyword NoSymm in Gaussian) in all the calculations.

\section{Results and Discussion}

\section{Spin Trimers}

We have characterized a noncollinear spin configuration of the triangular $\mathrm{H}_{3} \mathrm{He}_{3}$ test molecule, which is equivalent to a $\mathrm{C}_{3 h}$ spin trimer with $\mathrm{S}=1 / 2$ at each center. Figure 1 (a) shows the $\mathrm{H}_{3} \mathrm{He}_{3}$ structure with the hydrogens at the vertices of the triangle of side $3 \AA$, and the He atoms on the midpoint of each side, giving place to an effective antiferromagnetic superexchange coupling between the $\mathrm{H}$ atoms. The ground state of $\mathrm{H}_{3} \mathrm{He}_{3}$ is composed of two degenerate doublets $(\mathrm{S}=1 / 2)$ that can be described by four degenerate spin wave functions. 
Four different compromised noncollinear spin arrangements can be obtained from linear combinations of these degenerate spin wave functions. ${ }^{50}$ The scheme in Figure 1(a) shows one of these noncollinear spin arrangements obtained in the present calculation, where all the spin moments are of equal magnitude and lie on the plane of the molecule, and the spin-spin angle between neighboring spins of $120^{\circ}$ (it should be mentioned that in the absence of spin-orbit, as it is the case here, the total magnetization can be rigidly rotated without changing the total electronic energy). Table 1 summarizes the total and local spins calculated using different density functional approximations. It has been noted previously that the local spins obtained using the projection operators scheme show basis-set and functional dependence. ${ }^{29}$ For this particular system, however, the functional dependence of the local spins is very small in this case, and in all cases the local spin at each $\mathrm{H}$ center is reasonably close to the ideal value of 0.75 for a spin- $1 / 2$ center, as expected, due to the localized nature of the magnetic moments.

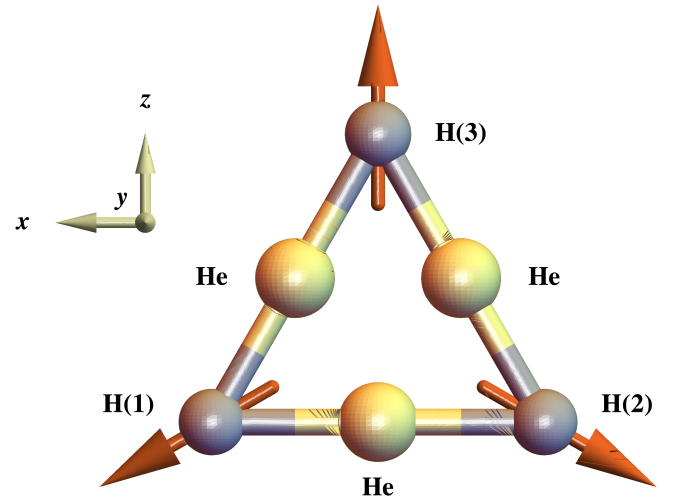

(a)

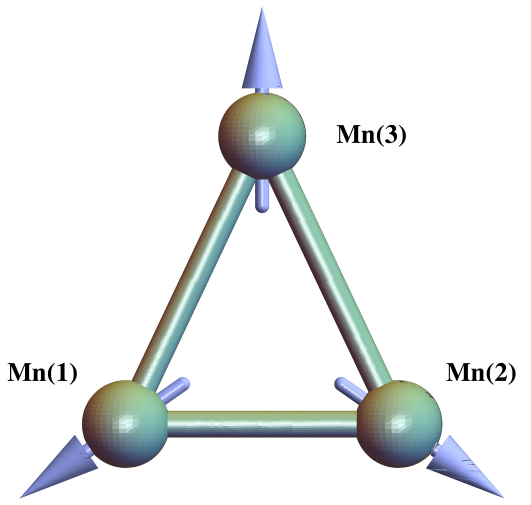

(b)

Figure 1: Schematic representation of the $\mathrm{H}_{3} \mathrm{He}_{3}$ molecule (a) and $\left[\mathrm{Mn}_{3}\right]$ core (b) with their respective noncollinear spin configurations shown as arrows.

For the $\left[\mathrm{Mn}_{3}\right]$ complex considered in our test calculations, we have used the relaxed 
Table 1: Calculated total and local spin values for $\mathrm{H}_{3} \mathrm{He}_{3} \cdot\left\langle\hat{\mathbf{S}}_{\mathrm{H}} \cdot \hat{\mathbf{S}}_{\mathrm{H}^{\prime}}\right\rangle$ refers to the off-diagonal terms in the local spin analysis.

\begin{tabular}{|l|c|c|c|c|}
\hline \hline Method & $\left\langle\hat{\mathbf{S}}^{2}\right\rangle$ & $\left|\left\langle\hat{\mathbf{S}}_{\mathrm{H}}\right\rangle\right|$ & $\left\langle\hat{\mathbf{S}}_{\mathrm{H}}^{2}\right\rangle$ & $\left\langle\hat{\mathbf{S}}_{\mathrm{H}} \cdot \hat{\mathbf{S}}_{\mathrm{H}^{\prime}}\right\rangle$ \\
\hline LSDA & 1.45 & 0.45 & 0.71 & -0.11 \\
PBE & 1.48 & 0.46 & 0.72 & -0.11 \\
BP86 & 1.47 & 0.46 & 0.72 & -0.11 \\
B3LYP & 1.48 & 0.46 & 0.72 & -0.11 \\
PBEh & 1.48 & 0.47 & 0.73 & -0.11 \\
\hline \hline
\end{tabular}

structure from the work of Luo et al. ${ }^{14}$ to calculate local spins for both the noncollinear and ncollinear spin cases (Supporting Information). Considering the $\mathrm{C}_{2 h}$ core structure of this complex, the three $\mathrm{Mn}$ are located at the vertices of an isosceles triangle, shown in Figure 1(b). In the actual structure of the complex, $\operatorname{Mn}(1)$ and $\operatorname{Mn}(2)$ are coupled with $\operatorname{Mn}(3)$ by a O-Mn-O linkage. As it can be expected, most of the molecular spin is localized at the three Mn centers. The discussion here is focused on the $\left\langle\hat{\mathbf{S}}_{A} \cdot \hat{\mathbf{S}}_{B}\right\rangle$ values pertinent to the three Mn centers. Table 2 summarizes the total and local noncollinear spins calculated using different DFT approximations. $\mathrm{Mn}(1)$ and $\mathrm{Mn}(2)$, which are closer to each other than to $\mathrm{Mn}(3)$, are equivalent in this noncollinear solution and have the same local spin values. From the local spin values $\left\langle\hat{\mathbf{S}}_{\mathrm{Mn}}^{2}\right\rangle$, it can be easily confirmed that the Mn centers are all

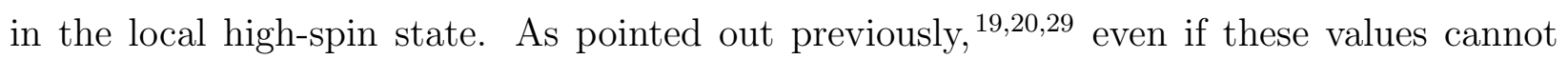
be formally interpreted as $\mathrm{S}(\mathrm{S}+1)$, it is interesting to note that the $\left\langle\hat{\mathbf{S}}_{\mathrm{Mn}(3)}^{2}\right\rangle$, except for PBEh, are close to the ideal value of 3.75 for a spin-3/2 center. The corresponding values for the other two centers, $\operatorname{Mn}(1)$ and $\operatorname{Mn}(2)$, are indeed noticeably different from the ideal $\mathrm{S}(\mathrm{S}+1)$. It can also be observed in Table 2 that both, the total and local values $\left\langle\hat{\mathbf{S}}_{\mathrm{Mn}}^{2}\right\rangle$, show a systematic increase with the incorporation of Hartree-Fock exchange, as expected from the higer electron localization of the $d$ electrons. The off-diagonal terms $\left\langle\hat{\mathbf{S}}_{A} \cdot \hat{\mathbf{S}}_{B}\right\rangle_{A \neq B}$, which indicate the presence and nature of the spin-spin coupling between the local spins, are all negative, corresponding to an antiferromagnetic arrangement between neighboring spins. The different functionals tested in this work give very similar values for the spin-spin 
angles $\left(\theta_{12} \approx 150^{\circ}\right.$ and $\theta_{13} \approx \theta_{23} \approx 105^{\circ}$, as calculated from $\hat{\mathbf{S}}_{\mathrm{Mn}(3)}$ (not shown here); however, the off-diagonal local spin values reflect a decrease of the antiferromagnetic $\operatorname{Mn}(1)-\operatorname{Mn}(2)$ interaction and an increase of the $\operatorname{Mn}(1,2)-\mathrm{Mn}(3)$ antiferromagnetic interaction with hybrid functionals. This can be interpreted as originated in an decreased metal-ligand interaction, as quantified by $\left\langle\hat{\mathbf{S}}_{\mathrm{Mn}} \cdot \hat{\mathbf{S}}_{\mathrm{L}}\right\rangle$ in Table 2. It is interesting to note that the local spin at the non-metal centers $\left\langle\hat{\mathrm{S}}_{\mathrm{L}}^{2}\right\rangle$, remains almost constant for all DFT approximations tested here.

The calculated total and local spin values for the collinear spin case corresponding to one of the broken-symmetry solutions with magnetization $\uparrow$ at $\operatorname{Mn}(1)$ and $\downarrow$ at $\operatorname{Mn}(2)$ and $\operatorname{Mn}(3)$ are summarized in Table 3. For this broken-symmetry solution, the calculated $\left\langle\hat{\mathbf{S}}_{\mathrm{Mn}(1,2)}^{2}\right\rangle$ follow a similar trend as in the noncollinear spin case, while $\left\langle\hat{\mathbf{S}}_{\mathrm{Mn}(3)}^{2}\right\rangle$ remains almost constant for the five funcionals tested here. This can be explained from the competing ferromagnetic $\left\langle\hat{\mathbf{S}}_{\mathrm{Mn}(2)} \cdot \hat{\mathbf{S}}_{\mathrm{Mn}(3)}\right\rangle$ and antiferromagnetic $\left\langle\hat{\mathbf{S}}_{\mathrm{Mn}(1)} \cdot \hat{\mathbf{S}}_{\mathrm{Mn}(3)}\right\rangle$ interactions. As Hartree-Fock exchange is incorporated, the magnetic exchange coupling between Mn centers becomes weaker. This effectively increases the magnitude of the metal $\left\langle\hat{\mathbf{S}}_{A} \cdot \hat{\mathbf{S}}_{B}\right\rangle$ in the collinear spin case, while in the noncollinear (frustrated) spin case, this is not possible for all metal pairs.

Table 2: Calculated total and local spin values for the $\left[\mathrm{Mn}_{3}\right]$ complex (noncollinear spin case). The indices label Mn centers as shown in Fig. 1. $\left\langle\hat{\mathbf{S}}_{\mathrm{Mn}} \cdot \hat{\mathbf{S}}_{\mathrm{L}}\right\rangle$ stands for the sum of the contributions of all three Mn centers and all non-metal atoms, and $\left\langle\hat{\mathrm{S}}_{\mathrm{L}}^{2}\right\rangle$ for all non-metal atoms.

\begin{tabular}{|l|c|c|c|c|c|c|c|}
\hline \hline Method & $\left\langle\hat{\mathrm{S}}^{2}\right\rangle$ & $\left\langle\hat{\mathrm{S}}_{1,2}^{2}\right\rangle$ & $\left\langle\hat{\mathrm{S}}_{3}^{2}\right\rangle$ & $\left\langle\hat{\mathbf{S}}_{1} \cdot \hat{\mathbf{S}}_{2}\right\rangle$ & $\left\langle\hat{\mathbf{S}}_{1,2} \cdot \hat{\mathbf{S}}_{3}\right\rangle$ & $\left\langle\hat{\mathbf{S}}_{\mathrm{Mn}} \cdot \hat{\mathbf{S}}_{\mathrm{L}}\right\rangle$ & $\left\langle\hat{\mathrm{S}}_{\mathrm{L}}^{2}\right\rangle$ \\
\hline LSDA & 4.85 & 4.32 & 3.64 & -1.28 & -0.30 & -11.48 & 7.81 \\
BP86 & 5.26 & 4.50 & 3.72 & -1.38 & -0.34 & -11.22 & 7.87 \\
PBE & 5.40 & 4.59 & 3.79 & -1.43 & -0.37 & -11.17 & 7.92 \\
B3LYP & 7.02 & 5.38 & 3.95 & -0.79 & -0.96 & -9.72 & 7.42 \\
PBEh & 8.84 & 5.69 & 4.17 & -0.52 & -1.17 & -8.80 & 7.82 \\
\hline \hline
\end{tabular}


Table 3: Calculated total and local spin values for the $\left[\mathrm{Mn}_{3}\right]$ complex (collinear spin case). The indices label Mn centers as shown in Fig. 1. $\left\langle\hat{\mathbf{S}}_{\mathrm{Mn}} \cdot \hat{\mathbf{S}}_{\mathrm{L}}\right\rangle$ stands for the sum of the contributions of all three Mn centers and all non-metal atoms, and $\left\langle\hat{\mathrm{S}}_{\mathrm{L}}^{2}\right\rangle$ for all non-metal atoms.

\begin{tabular}{|l|c|c|c|c|c|c|c|c|c|}
\hline \hline Method & $\left\langle\hat{\mathrm{S}}^{2}\right\rangle$ & $\left\langle\hat{\mathrm{S}}_{1}^{2}\right\rangle$ & $\left\langle\hat{\mathrm{S}}_{2}^{2}\right\rangle$ & $\left\langle\hat{\mathrm{S}}_{3}^{2}\right\rangle$ & $\left\langle\hat{\mathbf{S}}_{1} \cdot \hat{\mathbf{S}}_{2}\right\rangle$ & $\left\langle\hat{\mathbf{S}}_{1} \cdot \hat{\mathbf{S}}_{3}\right\rangle$ & $\left\langle\hat{\mathbf{S}}_{2} \cdot \hat{\mathbf{S}}_{3}\right\rangle$ & $\left\langle\hat{\mathbf{S}}_{\mathrm{Mn}} \cdot \hat{\mathbf{S}}_{\mathrm{L}}\right\rangle$ & $\left\langle\hat{\mathrm{S}}_{\mathrm{L}}^{2}\right\rangle$ \\
\hline LSDA & 4.88 & 4.69 & 4.14 & 3.53 & -1.50 & -1.19 & 0.92 & -6.38 & 8.83 \\
BP86 & 5.18 & 4.73 & 4.27 & 3.61 & -1.57 & -1.26 & 1.02 & -6.41 & 9.01 \\
PBE & 5.27 & 4.84 & 4.32 & 3.69 & -1.63 & -1.33 & 1.07 & -6.42 & 9.06 \\
B3LYP & 5.68 & 5.09 & 4.92 & 3.53 & -1.97 & -1.37 & 1.27 & -5.53 & 7.33 \\
PBEh & 5.93 & 5.38 & 5.27 & 3.60 & -2.23 & -1.48 & 1.40 & -5.56 & 7.42 \\
\hline \hline
\end{tabular}

\section{Application: Magnetic Exchange Couplings}

As an application of the local noncollinear spin analysis developed in this work, in this Section we show how it can be used to calculate magnetic exchange coupling parameters from a single reference spin state without explicit use of nominal spin values. Assuming a pairwise interaction between spins, magnetic exchange interactions can be modeled by the Heisenberg-Dirac-van-Vleck (HDVV) spin Hamiltonian ${ }^{51}$ of the form

$$
\hat{H}_{H D V V}=-\sum_{\langle i, j\rangle} J_{i j} \hat{\mathbf{S}}_{i} \cdot \hat{\mathbf{S}}_{j}
$$

where $\hat{\mathbf{S}}_{k}$ is the local spin operator on magnetic center $k$. This model spin Hamiltonian considers the isotropic exchange interaction between local spins associated with localized unpaired electrons. Taking the second-order derivative of the expectation value of Eq. 15 with respect to the inter-spin angle $\theta$ for a dinuclear system with centers $A$ and $B$ in its high-spin (HS) state, we can write

$$
\left.\frac{d^{2}\left\langle\hat{H}_{H D V V}\right\rangle}{d \theta^{2}}\right|_{\theta=0^{\circ}}=-\left.J_{A B} \frac{d^{2}\left\langle\hat{\mathbf{S}}_{A} \cdot \hat{\mathbf{S}}_{B}\right\rangle}{d \theta^{2}}\right|_{\theta=0^{\circ}} .
$$


Assuming that the KS system behaves as the HDVV system upon differential rotations of the inter-spin angle, we can replace $\left\langle\hat{H}_{H D V V}\right\rangle$ with the KS energy in Eq. 16 to obtain

$$
J_{A B}=-\left.\frac{\left.\frac{d^{2} E_{K S}}{d \theta^{2}}\right|_{\theta=0^{\circ}}}{\frac{d^{2}\left\langle\hat{\mathbf{S}}_{A} \cdot \hat{\mathbf{S}}_{B}\right\rangle}{d \theta^{2}}}\right|_{\theta=0^{\circ}}
$$

where in this case $\theta$ is the angle between the local spin vectors $\hat{\mathbf{S}}_{A}$ and $\hat{\mathbf{S}}_{B}$ in the KS system. Eq. 17 provides a method to calculate $J_{A B}$ from second derivatives with respect to the interspin angle of the total energy and $\left\langle\hat{\mathbf{S}}_{A} \cdot \hat{\mathbf{S}}_{B}\right\rangle$. In previous works we have provided a similar

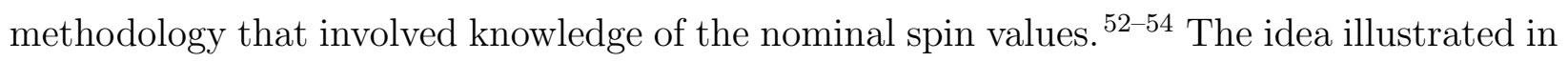
this Section provides one step further to the determination of $J_{A B}$ without external ad-hoc parameters.

To determine the derivatives in the right-hand-side of Eq. 17, we employ noncollinear constrained DFT as described in the Computational Details Section and implemented in Ref. 52. The angle between the local magnetic moments is introduced as a constraint in the energy expression and the generalized density is fully relaxed subject to that constraint. We scan the energy and $\left\langle\hat{\mathbf{S}}_{A} \cdot \hat{\mathbf{S}}_{B}\right\rangle$ landscapes for small angles and then perform a numerical fit to obtain the quadratic coefficients in a polynomial expansion. This strategy is not optimal for production calculations, where a method based on analytical derivatives would be desirable,, 53 but it serves our purposes in these proof-of-concept calculations. For comparision, the couplings $J_{A B}$ are also calculated using the conventional broken symmetry (BS) energy difference approach, $, 55,59$

$$
J_{A B}=\frac{E_{B S}-E_{H S}}{2 S_{A} S_{B}}
$$

where $E_{H S}$ and $E_{B S}$ are the energies of the high-spin (HS) and BS spin solutions, respectively.

To test the proposed methodology, we have selected the $\mathrm{H}-\mathrm{He}-\mathrm{H}$ linear molecule and the oxovanadium(IV) dimer $\left[\left(\mu-\mathrm{OCH}_{3}\right) \mathrm{VO}(\mathrm{ma})\right]_{2}\left(\mathrm{~V}_{2}\right.$ for short; see Supporting Information $)$ as benchmark systems. .60 For $\mathrm{H}-\mathrm{He}-\mathrm{H}$, we examine the performance of the proposed method 
at different strength of magnetic interactions corresponding to two different $\mathrm{H}-\mathrm{He}$ distances of $1.625 \AA$, and $2.0 \AA$. All the calculations in this section were carried out using Pople's style split-valence 6-311G** Gaussian basis ${ }^{6162}$ and the B3LYP exchange correlation functional. $\frac{4146}{49}$ All calculations use a tight convergence criterion of $10^{-8}$ Hartree in the energy. In Fig. 2 we show the KS energy and $\left\langle\hat{\mathbf{S}}_{\mathrm{H}(1)} \cdot \hat{\mathbf{S}}_{\mathrm{H}(2)}\right\rangle$ as a function of the angle $\theta$ for several different constraint angles about the $\mathrm{HS}$ spin state in $\mathrm{H}-\mathrm{He}-\mathrm{H}$. It is clear in this case that both quantities are close to a quadratic function in $\theta$, showing that the the contraint DFT method captures the physics of the HDVV model. Table 4 shows the exchange couplings
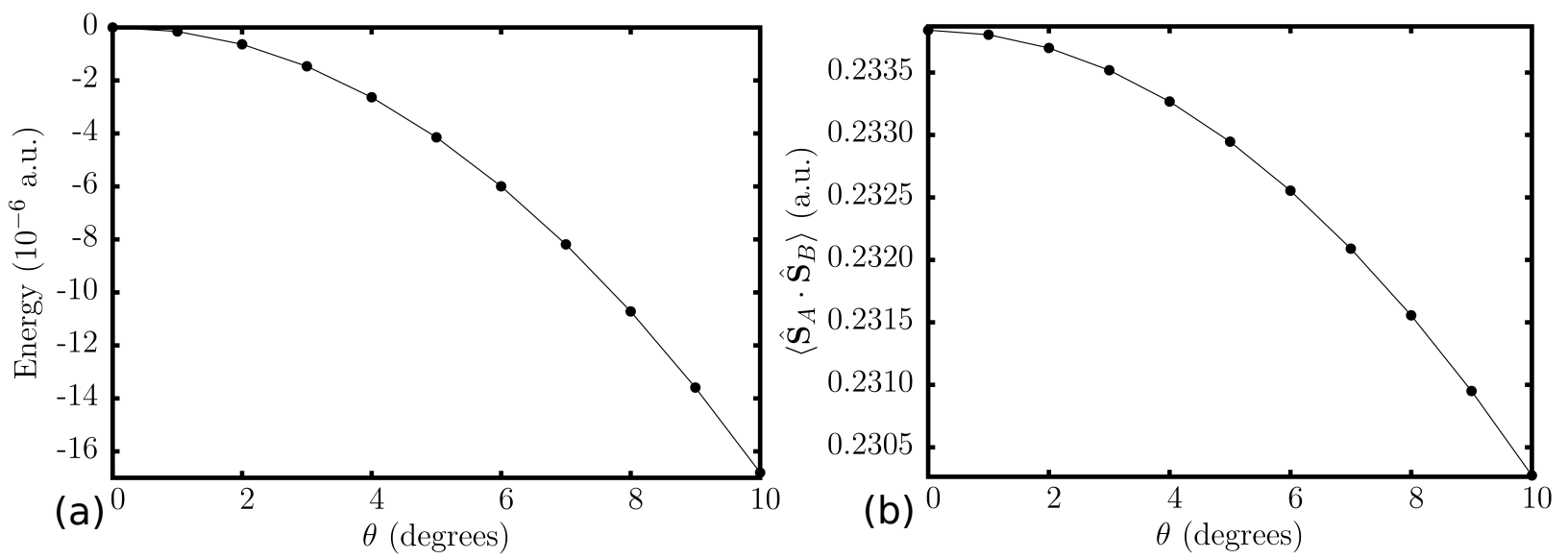

Figure 2: KS energy (a) and $\left\langle\hat{\mathbf{S}}_{\mathrm{H}(1)} \cdot \hat{\mathbf{S}}_{\mathrm{H}(2)}\right\rangle$ (b) variation as a function of the angle $\theta$ for the $\mathrm{H}-\mathrm{He}-\mathrm{H}$ molecule (H-He distance of $1.625 \AA$ ).

$J_{H H}$ obtained from second derivatives (Eq. 17) and from the BS energy differences approach. It is worth stressing that, although both methods give close $J$ values, the second derivatives method does not involve ad-hoc parameters, while the BS method uses $S_{H}=1 / 2$ in this case (Eq. 18). We note that for the shortest $\mathrm{H}-\mathrm{He}$ distance the relative deviation between both methods is the largerst, with a percentage difference of about $2.5 \%$. However, this is a somewhat unrealistic proof-of-concept case, and in most cases of practical interest the strength of $J$ is much smaller. Also, it should be pointed out that both methods would exactly agree only in the case of a perfectly localized BS spin configuration.

The $\mathrm{V}_{2}$ complex shows a strong antiferromagnetic coupling of about $-107 \mathrm{~cm}^{-1}$, as mea- 
Table 4: Calculated magnetic exchange couplings $\left(\right.$ in $\mathrm{cm}^{-1}$ ) for $\mathrm{H}-\mathrm{He}-\mathrm{H}$ molecule obtained from the second derivatives $\left(J^{S D}\right)$ method, and the BS energy differences approach $\left(J^{B S}\right)$, and percentage deviation.

\begin{tabular}{lccc}
\hline \hline H-He distance & $J^{S D}$ & $J^{B S}$ & deviation (\%) \\
\hline $2.00 \AA$ & -113.8 & -113.9 & $0.1 \%$ \\
$1.625 \AA$ & -1051.0 & -1025.6 & $2.5 \%$ \\
\hline \hline
\end{tabular}

sured by temperature-dependent magnetic susceptibility experiments. ${ }^{60}$ For this complex, using the same procedure described above, we obtained a couplings of $-179 \mathrm{~cm}^{-1}$ with the second derivative approach proposed in this work, and $-201 \mathrm{~cm}^{-1}$ for the BS energy differences approach. We found that the small deviation between both methodologies is encouraging, especially considering that they are not expected to yield identical values for realistic systems such as the $\mathrm{V}_{2}$ complex. As mentioned before, from a practical viewpoint a methodology that employs an analytical linear response implementation of this method would be desirable to extract both derivatives in Eq. 17. Work along this line is in progress.

\section{Concluding Remarks}

In this work we have derived the expressions for the local spin analysis for the case of a general noncollinear spin single-reference state. This analysis is based on the decomposition of the expectation value of the square of the total spin operator and utilizes general orthogonal atomic projectors. We have also implemented this decomposition using Löwdin projection operators and showed its applicability to characterize the local spins of two prototypical cases, $\mathrm{H}_{3} \mathrm{He}_{3}$ and the a $\left[\mathrm{Mn}_{3}\right]$ complex, where spin noncollinearity arises from geometrical frustration of antiferromagnetic interactions. For both systems, the expected compromised noncollinear spin arrangements are predicted by all the density functional approximations tested here. While for $\mathrm{H}_{3} \mathrm{He}_{3}$ the local spin values at each $\mathrm{H}$ center are essentially the same for all the functionals, the $\left[\mathrm{Mn}_{3}\right]$ complex shows a strong dependence on the functionals used. 
We want to stress that the local spin analysis requires minimal additional computational resources and can be readily incorporated to any electronic structure code with noncollinear spin capabilities. Besides its potential as an analysis tool, as a practical application we provide a methodology that utilizes the local spin decomposition for the determination of magnetic exchange couplings in magnetic molecules. With this method, we evaluate magnetic exchange couplings from second derivatives of the KS energy and local spin-spin pair

correlation values $\left\langle\hat{\mathbf{S}}_{A} \cdot \hat{\mathbf{S}}_{B}\right\rangle$ with respect to the interatomic spin angles. Our calculations on the benckmark cases $\mathrm{H}-\mathrm{He}-\mathrm{H}$ and the oxovanadium(IV) dimer $\left[\left(\mu-\mathrm{OCH}_{3}\right) \mathrm{VO}(\mathrm{ma})\right]_{2}$ show that our approach to calculate magnetic exchange couplings using noncollinear local spin analysis yields couplings comparable to the ones obtained from energy difference methods. However, in contrast to traditional energy difference based methods, our approach does not require any a priori knowledge of the nominal spin values, providing a route to the blackbox calculation of this property.

\section{Acknowledgements}

This work was supported by the Office of Basic Energy Sciences, US Department of Energy, de-sc0005027. JEP thanks Professor Pedro Salvador for helpful comments and suggestions.

\section{Supporting Information}

The Supporting Information is available free of charge on the ACS Publications website at DOI:. Complete derivation of Eq. 10 and 11, and Cartesian corrdinates of the $\left[\mathrm{Mn}_{3}\right]$ complex and oxovanadium(IV) dimer. 


\section{References}

(1) Ma, P.-W.; Dudarev, S. L. Constrained Density Functional for Non-Collinear Magnetism. Phys. Rev. B 2015, 91, 054420.

(2) Peralta, J. E.; Scuseria, G. E.; Frisch, M. J. Noncollinear Magnetism in Density Functional Calculations. Phys. Rev. B 2007, 75, 125119.

(3) Sticht, J.; Höck, K.-H.; Kübler, J. Non-Collinear Itinerant Magnetism: the Case of $\mathrm{Mn}_{3}$ Sn. J. Phys. Condens. Matter 1989, 1, 8155-8170.

(4) Delyagin, N.; Erzinkyan, A.; Parfenova, V.; Rozantsev, I. Mössbauer Study of Local Environment Effects in the Ordered $\mathrm{Fe}_{70} \mathrm{Al}_{30}$ Invar Alloy: Temperature Dependence of Isomer Shift in the Spin-Glass Phase. J. Magn. Magn. Mater. 2011, 323, 3058-3062.

(5) Peters, L.; Ghosh, S.; Sanyal, B.; van Dijk, C.; Bowlan, J.; de Heer, W.; Delin, A.; Di Marco, I.; Eriksson, O.; Katsnelson, M. I.; Johansson, B.; Kirilyuk, A. Magnetism and Exchange Interaction of Small Rare-Earth Clusters; Tb as a Representative. Sci. Rep. 2016, 6, 19676.

(6) Podewitz, M.; Herrmann, C.; Malassa, A.; Westrhausen, M.; Reiher, M. Spin-Spin Interactions in Polynuclear Transition-Metal Complexes. J. Chem. Phys. Lett. 2008, 451, 301-308.

(7) Cramer, C. J.; Truhlar, D. G. Density Functional Theory for Transition Metals and Transition Metal Chemistry. Phys. Chem. Chem. Phys. 2009, 11, 10757-10816.

(8) Capelle, K. A Bird's-Eye View of Density-Functional Thoery. Braz. J. Phys. 2006, 36, $1318-1343$.

(9) Jensen, F. Introduction to Computational Chemistry, 2nd ed.; John Wiley \& Sons: Chichester, 2007. 
(10) Szabo, A.; Ostlund, N. S. Modern Quantum Chemistry: Introduction to Advanced Electronic Structure Theory; Dover Publications: New York, 1996.

(11) Jacob, C. R.; Reiher, M. Spin in Density-Functional Theory. Int. J. Quantum chem. 2012, 112, 3661-3684.

(12) Kübler, J.; Höck, K.-H.; Sticht, J.; Williams, A. R. Density Functional Theory of NonCollinear Magnetism. J. Phys. F: Met. Phys. 1988, 18, 469-483.

(13) Peralta, J. E.; Scuseria, G. E. Relativistic All-electron Two-Component Self-Consistent Density Functional Calculations Including One-electron Scalar and Spin-Orbit Effects. J. Chem. Phys. 2004, 120, 5875-5881.

(14) Luo, S.; I. Rivalta, V. B.; Truhlar, D. G. Noncollinear Spins Provide a Self-Consistent Treatment of the Low-Spin State of a Biomimetic Oxomanganese Synthetic Trimer Inspired by the Oxygen Evolving Complex of Photosystem II. Phys. Chem. Lett. 2012, 2, 2629-2633.

(15) Scalmanni, G.; Frisch, M. J. A New Approach to Noncollinear Spin Density Functional Theory beyond the Local Density Approximation. J. Chem. Theory Comput. 2012, 8, 2193-2196.

(16) Bulik, I.; Scalmanni, G.; Frisch, M. J.; Scuseria, G. E. Noncollinear Density Functional Theory having Proper Invariance and Local Torque Properties. Phys. Rev. B 2013, 87, 035117.

(17) Mejía-López, J.; Romero, A. H.; Garcia, M. E.; Morán-López, J. L. Noncollinear Magnetism, Spin Frustration, and Magnetic Nanodomains in Small $\mathrm{Mn}_{n}$ Clusters. Phys. Rev. B 2006, 74, 140405.

(18) Peralta, J. E.; Hod, O.; Scuseria, G. E. Magnetization Dynamics from Time-Dependent 
Noncollinear Spin Density Functional Theory Calculations. J. Chem. Theory Comput. 2015, 11, 3661-3668.

(19) Clark, A.; Davidson, E. R. Local Spin. J. Chem. Phys. 2001, 115, 7382-7392.

(20) Reiher, M. On the Definition of Local Spin in Relativistic and Nonrelativistic Quantum Chemistry. Farad. Discuss. 2007, 135, 97-124.

(21) Mayer, I. Local Spins: An Alternative Treatment for Single Determinant Wave Functions. Chem. Phys. Lett. 2007, 440, 357-359.

(22) Manz, T. A.; Sholl, D. S. Methods for Computing Accurate Atomic Spin Moments for Collinear and Noncollinear Magnetism in Periodic and Nonperiodic Materials. $J$. Chem. Theory Comput. 2011, 7, 4146-4164.

(23) Alcoba, D. R.; Torre, A.; Lain, L.; Bochicchio, R. C. Determination of Local Spins by Means of a Spin-Free Treatment. J. Chem. Theory Comput. 2011, 7, 3560-3566.

(24) Schmidt, J. R.; Shenvi, N.; Tully, J. C. Controlling Spin Contamination using Constrained Density Functional Theory. J. Chem. Phys. 2008, 129, 114110.

(25) Wang, J.; Becke, A. D.; Jr., V. H. S. Evaluation of $\left\langle S^{2}\right\rangle$ in Restricted, Unrestricted Hartree-Fock, and Density Functional Based Theories. J. Chem. Phys. 1995, 102, 34773480.

(26) Cohen, A. J.; Tozer, D. J.; Handy, N. C. Evaluation of $\left\langle S^{2}\right\rangle$ in density functional theory. J. Chem. Phys. 2007, 126, 214104.

(27) Schmidt, J. R.; Shenvi, N.; Tully, J. C. Controlling Spin Contamination Using Constrained Density Functional Theory. J. Chem. Phys. 2008, 129, 114110.

(28) Clark, A.; Davidson, E. R. Population Analyses That Utilize Projection Operators. Int. J. Quantum chem. 2003, 93, 384-394. 
(29) Herrmann, C.; Reiher, M.; Hess, B. A. Comparative Analysis of Local Spin Definitions. J. Chem. Phys. 2005, 122, 034102.

(30) Mayer, I. Local Spins: An Improved Treatment for Correlated Wave Functions. J. Chem. Phys. Lett. 2009, 478, 323-326.

(31) Alcoba, D. R.; Lain, L.; Torre, A.; Bochicchio, R. C. Local Spin: A Treatment Beyond Single Determinant Wave Functions. J. Chem. Phys. Lett. 2009, 470, 136-139.

(32) Cordoba, E. R.; Matito, E.; Salvador, P.; Mayer, I. Local Spins: Improved Hilbert-Sapce Analysis. Phys. Chem. Chem. Phys. 2012, 14, 15291-15298.

(33) Cordoba, E. R.; Matito, E.; Mayer, I.; Salvador, P. Toward a Unique Definition of the Local Spin. Chem. Theory Comput. 2012, 8, 1270-1279.

(34) Cordoba, E. R.; Salvador, P.; Reiher, M. Local Spin Analysis and Chemical Bonding. Chem. Euro. J. 2013, 19, 15267-15275.

(35) Löwdin, P.-O. The Nonorthogonality Problem Connected with the use of Atomic Wave Functions in the Theory of Molecules and Crystals. J. Chem. Phys. 1950, 18, 365-375.

(36) Frisch, M. J.; Trucks, G. W.; Schlegel, H. B.; Scuseria, G. E.; Robb, M. A.; Cheeseman, J. R.; Scalmani, G.; Barone, V.; Mennucci, B.; Petersson, G. A.; Nakatsuji, H.; Caricato, M.; Li, X.; Hratchian, H. P.; Izmaylov, A. F.; Bloino, J.; Zheng, G.; Sonnenberg, J. L.; Liang, W.; Hada, M.; Ehara, M.; Toyota, K.; Fukuda, R.; Hasegawa, J.; Ishida, M.; Nakajima, T.; Honda, Y.; Kitao, O.; Nakai, H.; Vreven, T.; Montgomery, J. A.; Jr.,; Peralta, J. E.; Ogliaro, F.; Bearpark, M.; Heyd, J. J.; Brothers, E.; Kudin, K. N.; Staroverov, V. N.; Keith, T.; Kobayashi, R.; Normand, J.; Raghavachari, K.; Rendell, A.; Burant, J. C.; Iyengar, S. S.; Tomasi, J.; Cossi, M.; Rega, N.; Millam, J. M.; Klene, M.; Knox, J. E.; Cross, J. B.; Bakken, V.; Adamo, C.; Jaramillo, J.; Gomperts, R.; Stratmann, R. E.; Yazyev, O.; Austin, A. J.; Cammi, R.; 
Pomelli, C.; Ochterski, J. W.; Martin, R. L.; Morokuma, K.; Zakrzewski, V. G.; Voth, G. A.; Salvador, P.; Dannenberg, J. J.; Dapprich, S.; Parandekar, P. V.; Mayhall, N. J.; Daniels, A. D.; Farkas, O.; Foresman, J. B.; Ortiz, J. V.; Cioslowski, J.; Fox, D. J. Gaussian Develpment Version, Revision H.32. 2017; Gaussian, Inc., Wallingford, CT.

(37) Schäfer, A.; Huber, C.; Ahlrichs, R. Fully Optimized Contracted Gaussian Basis Sets of Triple Zeta Valence Quality for Atoms Li to Kr. J. Chem. Phys. 1994, 100, 5829-5835.

(38) Slater, J. The Self-consistent Field for Molecules and Solids. Quantum Theory of Molecules and Solids; McGraw-Hill, New York, 1974; Vol. 4.

(39) Vosko, S. H.; Wilk, L.; Nusair, M. Accurate Spin-Dependent Electron Liquid Correlation Energies for Local Spin Density Calculations: A Critical Analysis. Can. J. Phys $1980,58,1200$.

(40) Perdew, J. P.; Burke, K.; Ernzerhof, M. Generalized Gradient Approximation Made Simple. Phys. Rev. Lett. 1996, 77, 3865.

(41) Becke, A. D. Density-Functional Exchange-Energy Approximation with Correct Asymptotic Behavior. Phys. Rev. A 1988, 38, 3098-3100.

(42) Perdew, J. P. Density-Functional Approximation for the Correlation Energy of the Inhomogeneous Electron Gas. Phys. Rev. B 1986, 33, 8822-8824.

(43) Perdew, J. P.; Ernzerhof, M.; Burke, K. Rationale for Mixing Exact Exchange with Density Functional Approximations. J. Chem. Phys. 1997, 105, 9982.

(44) Ernzerhof, M.; Scuseria, G. E. Assessment of the Perdew-Burke-Ernzerhof ExchangeCorrelation Functional. J. Chem. Phys. 1999, 110, 5029.

(45) Adamo, C.; Barone, V. Toward Reliable Density Functional Methods without Adjustable Parameters: The PBE0 Model. J. Chem. Phys. 1999, 110, 6158. 
(46) Lee, C.; Yang, W.; Parr, R. G. Development of the Colle-Salvetti Correlation-Energy Formula into a Functional of the Electron Density. Phys. Rev. B 1988, 37, 785.

(47) Becke, A. D. Density-Functional Thermochemistry. III. The Role of Exact Exchange. J. Chem. Phys. 1993, 98, 5648.

(48) Stephens, P. J.; Devlin, F. J.; Chabalowski, C. F.; Frisch, M. J. Ab Initio Calculation of Vibrational Absorption and Circular Dichroism Spectra Using Density Functional Force Fields. J. Phys. Chem. 1994, 98, 11623.

(49) Hertwig, R. H.; Koch, W. On the Parameterization of the Local Correlation Functional. What is Becke-3-LYP? Chem. Phys. Lett. 1997, 268, $345-351$.

(50) Dai, D.; Whangbo, M. H. Classical Spin and Quantum-Mechanical Descriptions of Geometric Spin Frustration. J. Chem. Phys. 2004, 121, 672-680.

(51) Heisenberg, W. Zur Theorie des Ferromagnetismus. Z. Phys. 1928, 49, 619-636.

(52) Peralta, J. E.; Barone, V. Magnetic Exchange Couplings from Noncollinear Spin Density Functional Perturbation Theory. J. Chem. Phys. 2008, 129, 194107.

(53) Phillips, J. J.; Peralta, J. E. Towards the Blackbox Computation of Magnetic Exchange Coupling Parameters in Polynuclear Transition-Metal Complexes: Theory, Implementation, and Application. J. Chem. Phys. 2013, 138, 174115.

(54) Joshi, R. P.; Phillips, J. J.; Peralta, J. E. Magnetic Exchange Couplings in Heterodinuclear Complexes Based on Differential Local Spin Rotations. J. Chem. Theory Comput. 2016, 12, 1728-1734.

(55) Ruiz, E.; Rodríguez-Fortea, A.; Cano, J.; Alvarez, S.; Alemany, P. About the Calculation of Exchange Coupling Constants in Polynuclear Transition Metal Complexes. J. Comput. Chem. 2003, 24, 982-989. 
(56) Ruiz, E.; Cano, J.; Alvarez, S.; Alemany, P. Broken Symmetry Approach to Calculation of Exchange Coupling Constants for Homobinuclear and Heterobinuclear Transition Metal Complexes. J. Comput. Chem. 1999, 20,1391-1400.

(57) Nishino, M.; Yamanaka, S.; Yoshioka, Y.; Yamaguchi, K. Theoretical Approaches to Direct Exchange Couplings between Divalent Chromium Ions in Naked Dimers, Tetramers, and Clusters. J. Phys. Chem. A 1997, 101, 705-712.

(58) Illas, F.; de P. R. Moreira, I.; Bofill, J.; Filatov, M. Extent and Limitations of DensityFunctional Theory in Describing Magnetic Systems. Phys. Rev. B 2004, 70, 132414.

(59) Dai, D.; Whangbo, M.-H. Spin Exchange Interactions of a Spin Dimer: Analysis of Broken-Symmetry Spin States in terms of the Eigenstates of Heisenberg and Ising Spin Hamiltonians. J. Chem. Phys. 2003, 118, 29-39.

(60) Sun, Y.; Melchior, M.; Summers, D. A.; Thompson, R. C.; Rettig, S. J.; Orvig, C. $\left[\left(\mu-\mathrm{OCH}_{3}\right) \mathrm{VO}(\mathrm{ma})\right](2)$, A Strongly Antiferromagnetic Oxovanadium(IV) Dimer. Inorg. Chem. 1998, 37, 3119.

(61) Krishnan, R.; Binkley, J. S.; Seeger, R.; Pople, J. A. Self-Consistent Molecular Orbital Methods. XX. A Basis Set for Correlated Wave Functions. J. Chem. Phys. 1980, 72, 650.

(62) Blaudeau, J.-P.; McGrath, M. P.; Curtiss, L. A.; Radom, L. Extension of Gaussian-2 (G2) Theory to Molecules Containing Third-Row Atoms K and Ca. J. Chem. Phys. 1997, 107, 5016-5021. 


\title{
Supporting information for:
}

\section{Local Noncollinear Spin Analysis}

\author{
Bayileyegn A. Abate, Rajendra P. Joshi, and Juan E. Peralta* \\ Department of Physics and Science of Advanced Materials, Central Michigan University, Mount \\ Pleasant, MI, 48859, USA \\ E-mail: peral1j@cmich.edu
}

${ }^{*}$ To whom correspondence should be addressed 


\section{Derivation of Eq. 10 and Eq. 11 from the main text}

Starting form Eq. 8 from the main text,

$$
\begin{aligned}
\left\langle\hat{\mathbf{S}}_{A} \cdot \hat{\mathbf{S}}_{B}\right\rangle= & \frac{3}{4} \delta_{A B} \sum_{m}\left\langle m\left|\hat{P}_{A}\right| m\right\rangle+ \\
& \frac{1}{2} \sum_{m, n}\left[\left\langle m n\left|\hat{\mathbf{S}}_{1} \cdot \hat{\mathbf{S}}_{2}\left[\hat{P}_{A} \hat{P}_{B}+\hat{P}_{B} \hat{P}_{A}\right]\right| m n\right\rangle-\right. \\
& \left.\left\langle m n\left|\hat{\mathbf{S}}_{1} \cdot \hat{\mathbf{S}}_{2}\left[\hat{P}_{A} \hat{P}_{B}+\hat{P}_{B} \hat{P}_{A}\right]\right| n m\right\rangle\right]
\end{aligned}
$$

where $m$ and $n$ label two-component spinors, which can be expressed as $|m\rangle=\left|m^{\uparrow}\right\rangle+\left|m^{\downarrow}\right\rangle$ and $|n\rangle=\left|n^{\uparrow}\right\rangle+\left|n^{\downarrow}\right\rangle$. Using this, we rewrite Eq. 1 as,

$$
\begin{aligned}
& \left\langle\hat{\mathbf{S}}_{A} \cdot \hat{\mathbf{S}}_{B}\right\rangle=\frac{3}{4} \delta_{A B}\left[\sum_{m}\left\langle m^{\uparrow}\left|\hat{P}_{A}\right| m^{\uparrow}\right\rangle+\sum_{m}\left\langle m^{\downarrow}\left|\hat{P}_{A}\right| m^{\downarrow}\right\rangle\right] \\
& +\frac{1}{2} \sum_{m, n}\left[\left\langle m^{\uparrow} n^{\uparrow}\left|\hat{\mathbf{S}}_{1} \cdot \hat{\mathbf{S}}_{2}\left[\hat{P}_{A} \hat{P}_{B}+\hat{P}_{B} \hat{P}_{A}\right]\right| m^{\uparrow} n^{\uparrow}\right\rangle-\left\langle m^{\uparrow} n^{\uparrow}\left|\hat{\mathbf{S}}_{1} \cdot \hat{\mathbf{S}}_{2}\left[\hat{P}_{A} \hat{P}_{B}+\hat{P}_{B} \hat{P}_{A}\right]\right| n^{\uparrow} m^{\uparrow}\right\rangle\right] \\
& +\frac{1}{2} \sum_{m, n}\left[\left\langle m^{\downarrow} n^{\downarrow}\left|\hat{\mathbf{S}}_{1} \cdot \hat{\mathbf{S}}_{2}\left[\hat{P}_{A} \hat{P}_{B}+\hat{P}_{B} \hat{P}_{A}\right]\right| m^{\downarrow} n^{\downarrow}\right\rangle-\left\langle m^{\downarrow} n^{\downarrow}\left|\hat{\mathbf{S}}_{1} \cdot \hat{\mathbf{S}}_{2}\left[\hat{P}_{A} \hat{P}_{B}+\hat{P}_{B} \hat{P}_{A}\right]\right| n^{\downarrow} m^{\downarrow}\right\rangle\right] \\
& +\frac{1}{2} \sum_{m, n}\left[\left\langle m^{\uparrow} n^{\downarrow}\left|\hat{\mathbf{S}}_{1} \cdot \hat{\mathbf{S}}_{2}\left[\hat{P}_{A} \hat{P}_{B}+\hat{P}_{B} \hat{P}_{A}\right]\right| m^{\uparrow} n^{\downarrow}\right\rangle-\left\langle m^{\uparrow} n^{\downarrow}\left|\hat{\mathbf{S}}_{1} \cdot \hat{\mathbf{S}}_{2}\left[\hat{P}_{A} \hat{P}_{B}+\hat{P}_{B} \hat{P}_{A}\right]\right| n^{\downarrow} m^{\uparrow}\right\rangle\right] \\
& +\frac{1}{2} \sum_{m, n}\left[\left\langle m^{\downarrow} n^{\uparrow}\left|\hat{\mathbf{S}}_{1} \cdot \hat{\mathbf{S}}_{2}\left[\hat{P}_{A} \hat{P}_{B}+\hat{P}_{B} \hat{P}_{A}\right]\right| m^{\downarrow} n^{\uparrow}\right\rangle-\left\langle m^{\downarrow} n^{\uparrow}\left|\hat{\mathbf{S}}_{1} \cdot \hat{\mathbf{S}}_{2}\left[\hat{P}_{A} \hat{P}_{B}+\hat{P}_{B} \hat{P}_{A}\right]\right| n^{\uparrow} m^{\downarrow}\right\rangle\right] .
\end{aligned}
$$

It is convenient at this point to label the terms containing $\hat{\mathbf{S}}_{1} \cdot \hat{\mathbf{S}}_{2}$ from $A-H$ :

$$
\left\langle\hat{\mathbf{S}}_{A} \cdot \hat{\mathbf{S}}_{B}\right\rangle=\frac{3}{4} \delta_{A B} X+\frac{1}{2}[(A-B)+(C-D)+(E-F)+(G-H)]
$$

Now, we evaluate each of these terms separately. To this end, we make use of the following relations: 


$$
\begin{array}{r}
\hat{\mathbf{S}}_{1} \cdot \hat{\mathbf{S}}_{2}=\hat{S}_{z}(1) \hat{S}_{z}(2)+\frac{1}{2}\left[\hat{S}^{+}(1) \hat{S}^{-}(2)+\hat{S}^{-}(1) \hat{S}^{+}(2)\right], \\
\hat{S}_{z}(1)\left|m^{\uparrow}\right\rangle=\frac{1}{2}\left|m^{\uparrow}\right\rangle, \\
\hat{S}_{z}(1)\left|m^{\downarrow}\right\rangle=-\frac{1}{2}\left|m^{\downarrow}\right\rangle, \\
\hat{S}^{+}(1)\left|m^{\uparrow}\right\rangle=0, \\
\hat{S}^{-}(1)\left|m^{\downarrow}\right\rangle=0, \\
\hat{S}^{-}(1)\left|m^{\uparrow}\right\rangle=\left|m^{\downarrow}\right\rangle, \quad \text { and } \\
\hat{S}^{+}(1)\left|m^{\downarrow}\right\rangle=\left|m^{\uparrow}\right\rangle .
\end{array}
$$

The first term is

$$
A=\sum_{m, n}\left\langle m^{\uparrow} n^{\uparrow}\left|\hat{\mathbf{S}}_{1} \cdot \hat{\mathbf{S}}_{2}\left[\hat{P}_{A} \hat{P}_{B}+\hat{P}_{B} \hat{P}_{A}\right]\right| m^{\uparrow} n^{\uparrow}\right\rangle
$$

which using Eq. 4 becomes

$$
\begin{aligned}
A= & \sum_{m, n}\left[\left\langle m^{\uparrow} n^{\uparrow}\left|\hat{S}_{z}(1) \hat{S}_{z}(2)\left[\hat{P}_{A} \hat{P}_{B}+\hat{P}_{B} \hat{P}_{A}\right]\right| m^{\uparrow} n^{\uparrow}\right\rangle\right. \\
& +\frac{1}{2}\left[\langle m ^ { \uparrow } n ^ { \uparrow } | \left[\hat{S}^{+}(1) \hat{S}^{-}(2)\left[\hat{P}_{A} \hat{P}_{B}+\hat{P}_{B} \hat{P}_{A}\right]\left|m^{\uparrow} n^{\uparrow}\right\rangle\right.\right. \\
& \left.\left.\left.+\left\langle m^{\uparrow} n^{\uparrow}\right| \hat{S}^{-}(1) \hat{S}^{+}(2)\right]\left[\hat{P}_{A} \hat{P}_{B}+\hat{P}_{B} \hat{P}_{A}\right]\left|m^{\uparrow} n^{\uparrow}\right\rangle\right]\right] .
\end{aligned}
$$

Simplifying, this gives

$$
A=\frac{1}{4} \sum_{m, n}\left\langle m^{\uparrow} n^{\uparrow}\left|\left[\hat{P}_{A} \hat{P}_{B}+\hat{P}_{B} \hat{P}_{A}\right]\right| m^{\uparrow} n^{\uparrow}\right\rangle+0+0 .
$$




$$
A=\frac{1}{2} \sum_{m, n}\left\langle m^{\uparrow}\left|\hat{P}_{A}\right| m^{\uparrow}\right\rangle\left\langle n^{\uparrow}\left|\hat{P}_{B}\right| n^{\uparrow}\right\rangle
$$

Similarly, the $B, C$, and $E$ terms are:

$$
\begin{aligned}
& B=\frac{1}{2} \sum_{m, n}\left\langle m^{\uparrow}\left|\hat{P}_{A}\right| n^{\uparrow}\right\rangle\left\langle n^{\uparrow}\left|\hat{P}_{B}\right| m^{\uparrow}\right\rangle \\
& C=\frac{1}{2} \sum_{m, n}\left\langle m^{\downarrow}\left|\hat{P}_{A}\right| m^{\downarrow}\right\rangle\left\langle n^{\downarrow}\left|\hat{P}_{B}\right| n^{\downarrow}\right\rangle \\
& D=\frac{1}{2} \sum_{m, n}\left\langle m^{\downarrow}\left|\hat{P}_{A}\right| n^{\downarrow}\right\rangle\left\langle n^{\downarrow}\left|\hat{P}_{B}\right| m^{\downarrow}\right\rangle
\end{aligned}
$$

The mixed-spin terms $(E-H)$ can also be reduced using Eq. 4:

$$
\begin{aligned}
E= & \sum_{m, n}\left[\left\langle m^{\uparrow} n^{\downarrow}\left|\hat{S}_{z}(1) \hat{S}_{z}(2)\left[\hat{P}_{A} \hat{P}_{B}+\hat{P}_{B} \hat{P}_{A}\right]\right| m^{\uparrow} n^{\downarrow}\right\rangle\right. \\
& +\frac{1}{2}\left[\langle m ^ { \uparrow } n ^ { \downarrow } | \left[\hat{S}^{+}(1) \hat{S}^{-}(2)\left[\hat{P}_{A} \hat{P}_{B}+\hat{P}_{B} \hat{P}_{A}\right]\left|m^{\uparrow} n^{\downarrow}\right\rangle\right.\right. \\
& \left.\left.\left.+\left\langle m^{\uparrow} n^{\downarrow}\right| \hat{S}^{-}(1) \hat{S}^{+}(2)\right]\left[\hat{P}_{A} \hat{P}_{B}+\hat{P}_{B} \hat{P}_{A}\right]\left|m^{\uparrow} n^{\downarrow}\right\rangle\right]\right]
\end{aligned}
$$

or

$$
\begin{gathered}
E=\sum_{m, n}\left[-\frac{1}{4}\left\langle m^{\uparrow} n^{\downarrow}\left|\left[\hat{P}_{A} \hat{P}_{B}+\hat{P}_{B} \hat{P}_{A}\right]\right| m^{\uparrow} n^{\downarrow}\right\rangle+0+\frac{1}{2} \sum_{m, n}\left\langle m^{\uparrow} n^{\downarrow}\left|\left[\hat{P}_{A} \hat{P}_{B}+\hat{P}_{B} \hat{P}_{A}\right]\right| m^{\downarrow} n^{\uparrow}\right\rangle\right] \\
=\sum_{m, n}\left[-\frac{1}{2}\left\langle m^{\uparrow}\left|\hat{P}_{A}\right| m^{\uparrow}\right\rangle\left\langle n^{\downarrow}\left|\hat{P}_{B}\right| n^{\downarrow}\right\rangle+0+\sum_{m, n}\left\langle m^{\uparrow}\left|\hat{P}_{A}\right| m^{\downarrow}\right\rangle\left\langle n^{\downarrow}\left|\hat{P}_{B}\right| n^{\uparrow}\right\rangle\right] .
\end{gathered}
$$

Similarly, the terms $F-H$ can be expressed as: 


$$
\begin{aligned}
& F=\sum_{m, n}\left[\left\langle m^{\uparrow} n^{\downarrow}\left|\hat{S}_{z}(1) \hat{S}_{z}(2)\left[\hat{P}_{A} \hat{P}_{B}+\hat{P}_{B} \hat{P}_{A}\right]\right| n^{\downarrow} m^{\uparrow}\right\rangle\right. \\
& +\frac{1}{2}\left[\langle m ^ { \uparrow } n ^ { \downarrow } | \left[\hat{S}^{+}(1) \hat{S}^{-}(2)\left[\hat{P}_{A} \hat{P}_{B}+\hat{P}_{B} \hat{P}_{A}\right]\left|n^{\downarrow} m^{\uparrow}\right\rangle\right.\right. \\
& \left.\left.+\left\langle m^{\uparrow} n^{\downarrow}\left|\hat{S}^{-}(1) \hat{S}^{+}(2)\left[\hat{P}_{A} \hat{P}_{B}+\hat{P}_{B} \hat{P}_{A}\right]\right| n^{\downarrow} m^{\uparrow}\right\rangle\right]\right] \\
& =\sum_{m, n}\left[-\frac{1}{2}\left\langle m^{\uparrow}\left|\hat{P}_{A}\right| n^{\downarrow}\right\rangle\left\langle n^{\downarrow}\left|\hat{P}_{B}\right| m^{\uparrow}\right\rangle\right. \\
& \left.+\left\langle m^{\uparrow}\left|\hat{P}_{A}\right| n^{\uparrow}\right\rangle\left\langle n^{\downarrow}\left|\hat{P}_{B}\right| m^{\downarrow}\right\rangle+0\right], \\
& G=\sum_{m, n}\left[\left\langle m^{\downarrow} n^{\uparrow}\left|\hat{S}_{z}(1) \hat{S}_{z}(2)\left[\hat{P}_{A} \hat{P}_{B}+\hat{P}_{B} \hat{P}_{A}\right]\right| m^{\downarrow} n^{\uparrow}\right\rangle\right. \\
& +\frac{1}{2}\left[\langle m ^ { \downarrow } n ^ { \uparrow } | \left[\hat{S}^{+}(1) \hat{S}^{-}(2)\left[\hat{P}_{A} \hat{P}_{B}+\hat{P}_{B} \hat{P}_{A}\right]\left|m^{\downarrow} n^{\uparrow}\right\rangle\right.\right. \\
& \left.\left.+\left\langle m^{\downarrow} n^{\uparrow}\left|\hat{S}^{-}(1) \hat{S}^{+}(2)\left[\hat{P}_{A} \hat{P}_{B}+\hat{P}_{B} \hat{P}_{A}\right]\right| m^{\downarrow} n^{\uparrow}\right\rangle\right]\right] \\
& =\sum_{m, n}\left[-\frac{1}{2}\left\langle m^{\downarrow}\left|\hat{P}_{A}\right| m^{\downarrow}\right\rangle\left\langle n^{\uparrow}\left|\hat{P}_{B}\right| n^{\uparrow}\right\rangle+0\right. \\
& \left.+\left\langle m^{\downarrow}\left|\hat{P}_{A}\right| m^{\uparrow}\right\rangle\left\langle n^{\uparrow}\left|\hat{P}_{B}\right| n^{\downarrow}\right\rangle\right],
\end{aligned}
$$

and 


$$
\begin{aligned}
H= & \sum_{m, n}\left[\left\langle m^{\downarrow} n^{\uparrow}\left|\hat{S}_{z}(1) \hat{S}_{z}(2)\left[\hat{P}_{A} \hat{P}_{B}+\hat{P}_{B} \hat{P}_{A}\right]\right| n^{\uparrow} m^{\downarrow}\right\rangle\right. \\
& +\frac{1}{2}\left[\langle m ^ { \downarrow } n ^ { \uparrow } | \left[\hat{S}^{+}(1) \hat{S}^{-}(2)\left[\hat{P}_{A} \hat{P}_{B}+\hat{P}_{B} \hat{P}_{A}\right]\left|n^{\uparrow} m^{\downarrow}\right\rangle\right.\right. \\
& \left.+\left\langle m^{\downarrow} n^{\uparrow}\left|\hat{S}^{-}(1) \hat{S}^{+}(2)\left[\hat{P}_{A} \hat{P}_{B}+\hat{P}_{B} \hat{P}_{A}\right]\right| n^{\uparrow} m^{\downarrow}\right\rangle\right] \\
= & \sum_{m, n}\left[-\frac{1}{2}\left\langle m^{\downarrow}\left|\hat{P}_{A}\right| n^{\uparrow}\right\rangle\left\langle n^{\uparrow}\left|\hat{P}_{B}\right| m^{\downarrow}\right\rangle+0\right. \\
& \left.+\left\langle m^{\downarrow}\left|\hat{P}_{A}\right| n^{\downarrow}\right\rangle\left\langle n^{\uparrow}\left|\hat{P}_{B}\right| m^{\uparrow}\right\rangle\right]
\end{aligned}
$$

Substituting $A$ through $H$ in Eq. 3, we obtain Eq. 9 from the manuscript:

$$
\begin{aligned}
\left\langle\hat{\mathbf{S}}_{A} \cdot \hat{\mathbf{S}}_{B}\right\rangle= & \frac{3}{4} \delta_{A B} \sum_{m}\left[\left\langle m^{\uparrow}\left|\hat{P}_{A}\right| m^{\uparrow}\right\rangle+\left\langle m^{\downarrow}\left|\hat{P}_{A}\right| m^{\downarrow}\right\rangle\right]+ \\
& \sum_{m, n}\left[\frac{1}{4}\left\langle m^{\uparrow}\left|\hat{P}_{A}\right| m^{\uparrow}\right\rangle\left\langle n^{\uparrow}\left|\hat{P}_{B}\right| n^{\uparrow}\right\rangle+\frac{1}{4}\left\langle m^{\downarrow}\left|\hat{P}_{A}\right| m^{\downarrow}\right\rangle\left\langle n^{\downarrow}\left|\hat{P}_{B}\right| n^{\downarrow}\right\rangle\right. \\
& -\frac{1}{4}\left\langle m^{\uparrow}\left|\hat{P}_{A}\right| n^{\uparrow}\right\rangle\left\langle n^{\uparrow}\left|\hat{P}_{B}\right| m^{\uparrow}\right\rangle-\frac{1}{4}\left\langle m^{\downarrow}\left|\hat{P}_{A}\right| n^{\downarrow}\right\rangle\left\langle n^{\downarrow}\left|\hat{P}_{B}\right| m^{\downarrow}\right\rangle \\
& -\frac{1}{4}\left\langle m^{\uparrow}\left|\hat{P}_{A}\right| m^{\uparrow}\right\rangle\left\langle n^{\downarrow}\left|\hat{P}_{B}\right| n^{\downarrow}\right\rangle-\frac{1}{4}\left\langle m^{\downarrow}\left|\hat{P}_{A}\right| m^{\downarrow}\right\rangle\left\langle n^{\uparrow}\left|\hat{P}_{B}\right| n^{\uparrow}\right\rangle \\
& -\frac{1}{2}\left\langle m^{\uparrow}\left|\hat{P}_{A}\right| n^{\uparrow}\right\rangle\left\langle n^{\downarrow}\left|\hat{P}_{B}\right| m^{\downarrow}\right\rangle-\frac{1}{2}\left\langle m^{\downarrow}\left|\hat{P}_{A}\right| n^{\downarrow}\right\rangle\left\langle n^{\uparrow}\left|\hat{P}_{B}\right| m^{\uparrow}\right\rangle \\
& +\frac{1}{2}\left\langle m^{\uparrow}\left|\hat{P}_{A}\right| m^{\downarrow}\right\rangle\left\langle n^{\downarrow}\left|\hat{P}_{B}\right| n^{\uparrow}\right\rangle+\frac{1}{2}\left\langle m^{\downarrow}\left|\hat{P}_{A}\right| m^{\uparrow}\right\rangle\left\langle n^{\uparrow}\left|\hat{P}_{B}\right| n^{\downarrow}\right\rangle \\
& \left.+\frac{1}{4}\left\langle m^{\uparrow}\left|\hat{P}_{A}\right| n^{\downarrow}\right\rangle\left\langle n^{\downarrow}\left|\hat{P}_{B}\right| m^{\uparrow}\right\rangle+\frac{1}{4}\left\langle m^{\downarrow}\left|\hat{P}_{A}\right| n^{\uparrow}\right\rangle\left\langle n^{\uparrow}\left|\hat{P}_{B}\right| m^{\downarrow}\right\rangle .\right],
\end{aligned}
$$

which can be expressed in terms of projected one-particle density matrices as: 


$$
\begin{aligned}
\left\langle\hat{\mathbf{S}}_{A} \cdot \hat{\mathbf{S}}_{B}\right\rangle= & \frac{3}{4} \boldsymbol{\delta}_{A B} \sum_{\mu \in A}\left[\mathbf{P}_{\mu \mu}^{\uparrow \uparrow}+\mathbf{P}_{\mu \mu}^{\downarrow \downarrow}\right]+\sum_{\mu \in A, v \in B}\left[\frac{1}{4} \mathbf{P}_{\mu \mu}^{\uparrow \uparrow} \mathbf{P}_{v v}^{\uparrow \uparrow}+\frac{1}{4} \mathbf{P}_{\mu \mu}^{\downarrow \downarrow} \mathbf{P}_{v v}^{\downarrow \downarrow}\right. \\
& -\frac{1}{4} \mathbf{P}_{\mu \nu}^{\uparrow \uparrow} \mathbf{P}_{v \mu}^{\uparrow \uparrow}-\frac{1}{4} \mathbf{P}_{\mu \nu}^{\downarrow \downarrow} \mathbf{P}_{v \mu}^{\downarrow \downarrow}-\frac{1}{4} \mathbf{P}_{\mu \mu}^{\uparrow \uparrow} \mathbf{P}_{v v}^{\downarrow \downarrow}-\frac{1}{4} \mathbf{P}_{\mu \mu}^{\downarrow \downarrow} \mathbf{P}_{v v}^{\uparrow \uparrow}-\frac{1}{2} \mathbf{P}_{\mu \nu}^{\uparrow \uparrow} \mathbf{P}_{v \mu}^{\downarrow \downarrow} \\
& \left.-\frac{1}{2} \mathbf{P}_{\mu \nu}^{\downarrow \downarrow} \mathbf{P}_{v \mu}^{\uparrow \uparrow}+\frac{1}{2} \mathbf{P}_{\mu \mu}^{\uparrow \downarrow} \mathbf{P}_{v v}^{\downarrow \uparrow}+\frac{1}{2} \mathbf{P}_{\mu \mu}^{\downarrow \uparrow} \mathbf{P}_{v v}^{\uparrow \downarrow}+\frac{1}{4} \mathbf{P}_{\mu v}^{\uparrow \downarrow} \mathbf{P}_{v \mu}^{\downarrow \uparrow}+\frac{1}{4} \mathbf{P}_{\mu \nu}^{\downarrow \uparrow} \mathbf{P}_{v \mu}^{\uparrow \downarrow}\right] .
\end{aligned}
$$

A more compact form of Eq. 13 can be derived by defining a vector $\overrightarrow{\mathbf{P}}_{\mu \nu}$ with cartesian components $\mathbf{P}_{\mu \nu}^{x}=\mathbf{P}_{\mu v}^{\uparrow \downarrow}+\mathbf{P}_{\mu v}^{\uparrow \downarrow}, \mathbf{P}_{\mu v}^{y}=i\left(\mathbf{P}_{\mu v}^{\uparrow \downarrow}-\mathbf{P}_{\mu v}^{\uparrow \downarrow}\right), \mathbf{P}_{\mu \nu}^{z}=\mathbf{P}_{\mu v}^{\uparrow \uparrow}-\mathbf{P}_{\mu \nu}^{\downarrow \downarrow}$, and scalor $\mathbf{P}_{\mu v}=\mathbf{P}_{\mu v}^{\uparrow \uparrow}+\mathbf{P}_{\mu \nu}^{\downarrow \downarrow}$. From these relations, we can write,

$$
\begin{aligned}
\mathbf{P}_{\mu v}^{\uparrow \downarrow} & =\frac{1}{2}\left(\mathbf{P}_{\mu v}^{x}-i \mathbf{P}_{\mu v}^{y}\right) \\
\mathbf{P}_{\mu v}^{\downarrow \uparrow} & =\frac{1}{2}\left(\mathbf{P}_{\mu v}^{x}+i \mathbf{P}_{\mu v}^{y}\right) \\
\mathbf{P}_{\mu v}^{\uparrow \uparrow} & =\frac{1}{2}\left(\mathbf{P}_{\mu v}+\mathbf{P}_{\mu v}^{z}\right) \\
\mathbf{P}_{\mu v}^{\downarrow \downarrow} & =\frac{1}{2}\left(\mathbf{P}_{\mu v}-\mathbf{P}_{\mu v}^{z}\right)
\end{aligned}
$$

Substituting these in Eq. 13 and working the algebra algebra, one obtains:

$$
\begin{aligned}
\left\langle\hat{\mathbf{S}}_{A} \cdot \hat{\mathbf{S}}_{B}\right\rangle= & \frac{3}{4} \delta_{A B} \mathbf{P}_{\mu \mu}+\frac{1}{4}\left[\frac{1}{2}\left(\mathbf{P}_{\mu \mu}^{z} \mathbf{P}_{v v}^{z}+\mathbf{P}_{\mu \mu} \mathbf{P}_{v v}\right)\right]-\frac{1}{4}\left[\frac{1}{2}\left(\mathbf{P}_{\mu \nu}^{z} \mathbf{P}_{v \mu}^{z}+\mathbf{P}_{\mu v} \mathbf{P}_{v \mu}\right)\right] \\
& -\frac{1}{4}\left[\frac{1}{2}\left(-\mathbf{P}_{\mu \mu}^{z} \mathbf{P}_{v v}^{z}+\mathbf{P}_{\mu \mu} \mathbf{P}_{v v}\right)\right]-\frac{1}{2}\left[\frac{1}{2}\left(-\mathbf{P}_{\mu \nu}^{z} \mathbf{P}_{v \mu}^{z}+\mathbf{P}_{\mu \nu} \mathbf{P}_{v \mu}\right)\right]+ \\
& \frac{1}{2}\left[\frac{1}{2}\left(\mathbf{P}_{\mu \mu}^{x} \mathbf{P}_{v v}^{x}+\mathbf{P}_{\mu \mu}^{y} \mathbf{P}_{v v}^{y}\right)\right]+\frac{1}{4}\left[\frac{1}{2}\left(\mathbf{P}_{\mu \nu}^{x} \mathbf{P}_{v \mu}^{x}+\mathbf{P}_{\mu \nu}^{y} \mathbf{P}_{v \mu}^{y}\right)\right],
\end{aligned}
$$

where we have dropped the summations for simplicity. Combining terms we have 


$$
\begin{aligned}
\left\langle\hat{\mathbf{S}}_{A} \cdot \hat{\mathbf{S}}_{B}\right\rangle & =\frac{3}{4} \boldsymbol{\delta}_{A B} \mathbf{P}_{\mu \mu}+\frac{1}{4}\left[\mathbf{P}_{\mu \mu}^{x} \mathbf{P}_{v v}^{x}+\mathbf{P}_{\mu \mu}^{y} \mathbf{P}_{v v}^{y}+\mathbf{P}_{\mu \mu}^{z} \mathbf{P}_{v v}^{z}\right] \\
& +\frac{1}{8}\left[\mathbf{P}_{\mu \nu}^{x} \mathbf{P}_{v \mu}^{x}+\mathbf{P}_{\mu \nu}^{y} \mathbf{P}_{v \mu}^{y}+\mathbf{P}_{\mu \nu}^{z} \mathbf{P}_{v \mu}^{z}\right]-\frac{3}{8} \mathbf{P}_{\mu v} \mathbf{P}_{\mu v}
\end{aligned}
$$

and rewritting this expression as

$$
\left\langle\hat{\mathbf{S}}_{A} \cdot \hat{\mathbf{S}}_{B}\right\rangle=\frac{3}{4}\left(\delta_{A B} \mathbf{P}_{\mu \mu}-\frac{1}{2} \mathbf{P}_{\mu \nu} \mathbf{P}_{v \mu}\right)+\frac{1}{4}\left(\overrightarrow{\mathbf{P}}_{\mu \mu} \cdot \overrightarrow{\mathbf{P}}_{v v}+\frac{1}{2} \overrightarrow{\mathbf{P}}_{\mu \nu} \cdot \overrightarrow{\mathbf{P}}_{v \mu}\right)
$$

Replacing the notation $\sum_{\mu \in A}$ and $\sum_{v \in B}$ with subindices $A$ and $B$, we obtain Eq. 11 of the main text:

$$
\left\langle\hat{\mathbf{S}}_{A} \cdot \hat{\mathbf{S}}_{B}\right\rangle=\frac{3}{4}\left(\delta_{A B} \mathbf{P}_{A A}-\frac{1}{2} \mathbf{P}_{A B} \mathbf{P}_{B A}\right)+\frac{1}{4}\left(\overrightarrow{\mathbf{P}}_{A A} \cdot \overrightarrow{\mathbf{P}}_{B B}+\frac{1}{2} \overrightarrow{\mathbf{P}}_{A B} \cdot \overrightarrow{\mathbf{P}}_{B A}\right) .
$$




\section{xyz Coordinates of the $\left[\left(\mathrm{Mn}^{4}\right)_{3} \mathrm{O}_{4} \mathrm{~L}_{4}\left(\mathrm{H}_{2} \mathrm{O}\right)\right]$ complex}

$$
\begin{aligned}
& \text { Mn }-1.3374750 .000000 \quad 0.000000 \\
& \text { Mn } 1.342218 \quad 0.000000 \quad 0.000000 \\
& \text { Mn } 0.000000 \quad 0.000000-2.982529 \\
& 00.0340521 .2006110 .192426 \\
& 0 \quad-0.029047-1.199108 \quad 0.200738 \\
& 0-1.3240970 .032363-1.806643 \\
& 01.324148-0.034859-1.807201 \\
& \begin{array}{llll}
0 & -2.850328 & 1.523388 & -0.183803
\end{array} \\
& \text { H }-3.562810 \quad 1.373748-0.840551 \\
& \mathrm{H}-3.287200 \quad 1.9383330 .590868 \\
& \text { o } 2.843692-1.538262-0.192931 \\
& \text { H } 3.570948-1.380000-0.831336 \\
& \text { H } 3.262879-1.970666 \quad 0.582092 \\
& N-2.857627-1.442896 \quad 0.179206 \\
& \mathrm{~N}-1.820394 \quad 0.129792 \quad 2.044717 \\
& \text { C }-3.270353-1.6400161 .504506 \\
& \mathrm{H}-4.011974-2.409746 \quad 1.729511 \\
& \text { C }-2.749507-0.838609 \quad 2.450485 \\
& \text { H }-3.039811-0.9022193 .501678 \\
& \text { C }-3.384877-2.130296-0.774635 \\
& \text { H }-4.155178-2.885147-0.580671 \\
& \mathrm{H}-3.057163-1.942672-1.796437 \\
& \text { C }-1.3842821 .0195972 .871403 \\
& \mathrm{H}-1.725148 \quad 1.047152 \quad 3.913428 \\
& \mathrm{H}-0.672919 \quad 1.764035 \quad 2.510134
\end{aligned}
$$




$$
\begin{aligned}
& \text { N 2.871919 1.434365 } 0.168008 \\
& \text { N 1.831267 -0.123939 2.043028 } \\
& \text { C } 3.2908121 .6342161 .490941 \\
& \text { H } 4.038048 \quad 2.4001661 .710131 \\
& \text { C } 2.767952 \quad 0.8400512 .441857 \\
& \text { H } 3.061993 \quad 0.905955 \quad 3.491835 \\
& \text { C } 3.3998682 .114592-0.790656 \\
& \text { H } 4.175370 \quad 2.865587-0.602578 \\
& \text { H } 3.0667371 .925091-1.810354 \\
& \text { C } 1.392914-1.0076092 .875169 \\
& \text { H } 1.738621-1.033620 \quad 3.915614 \\
& \mathrm{H} \quad 0.675144 \quad-1.748772 \quad 2.519931 \\
& \text { N } 1.4070490 .151523-4.581475 \\
& \text { N } 0.1100452 .036789-3.199373 \\
& \text { C } 1.658844 \quad 1.495434 \quad-4.895271 \\
& \text { H } 2.360270 \quad 1.737623 \quad-5.697498 \\
& \text { C } 1.009147 \quad 2.443168 \quad-4.199592 \\
& \text { H } 1.143177 \quad 3.510146-4.391802 \\
& \text { C } 2.000949-0.772153-5.257841 \\
& \text { H } 2.698601-0.528333-6.067469 \\
& \text { H } 1.810909-1.821410-5.037435 \\
& \text { C }-0.520178 \quad 2.914752-2.494848 \\
& \mathrm{H}-0.367300 \quad 3.986422-2.666658 \\
& \mathrm{H}-1.2072342 .581040-1.720601 \\
& \mathrm{~N}-1.413015-0.150614-4.576487 \\
& \mathrm{~N}-0.109851-2.036405-3.201246 \\
& \text { C }-1.666070-1.494328-4.889981
\end{aligned}
$$




$$
\begin{array}{llll}
\text { H } & -2.370980 & -1.736097 & -5.689272 \\
\mathrm{C} & -1.013741 & -2.442396 & -4.197256 \\
\mathrm{H} & -1.148613 & -3.509296 & -4.389319 \\
\mathrm{C} & -2.010266 & 0.773481 & -5.249237 \\
\mathrm{H} & -2.714326 & 0.530267 & -6.053490 \\
\mathrm{H} & -1.819717 & 1.822585 & -5.028464 \\
\mathrm{C} & 0.525104 & -2.914375 & -2.501032 \\
\text { H } & 0.372591 & -3.986000 & -2.673493 \\
\text { H } & 1.216046 & -2.580441 & -1.730213
\end{array}
$$

\section{xyz Coordinates of the $\left(\mu-\mathrm{OCH}_{3} \mathrm{VO}(\mathrm{ma})\right]_{2}$ complex}

$$
\begin{aligned}
& \text { V } 0.9334231361 .16726372 \quad 0.372422819 \\
& \mathrm{~V}-0.933423136-1.16726372-0.372422819 \\
& 0-0.0700049576-0.330421 .15800644 \\
& 00.07000495760 .33042-1.15800644 \\
& \text { O } 5.90071107 \quad 1.569495 \quad 1.28536908 \\
& 02.39817005 \quad 0.787501 \quad 1.58345186 \\
& 02.456418421 .778761-0.79940382 \\
& 0 \quad 0.180746881 \quad 2.5089892 \quad 0.765079136 \\
& \text { C } 4.89661546 \quad 0.473602 \quad 3.14612825 \\
& \text { C } 4.7420872 \quad 1.1025014 \quad 1.82282137 \\
& \text { C } 3.57782962 \quad 1.2236554 \quad 1.12277847 \\
& \text { C } 3.58154222 \quad 1.7754568-0.175236544 \\
& \text { C } 4.810222962 .2600728-0.694623206 \\
& \text { C } 5.896278692 .1400202 \quad 0.053293588
\end{aligned}
$$




$$
\begin{aligned}
& \text { C }-0.032677831-0.7500534 \quad 2.51834785 \\
& \text { H } 4.78159995 \quad 0.958218 \quad 3.71248554 \\
& \text { H } 4.12440793-0.088112 \quad 3.40536994 \\
& \text { H } 5.61133587-0.088112 \quad 3.2879434 \\
& \text { H } 4.897638192 .577276-1.56267639 \\
& \text { H } 6.79481052 \quad 2.390038-0.171623419 \\
& \text { H }-0.704359343-1.189512 \quad 2.78210595 \\
& \text { H } 0.214483688 \quad 0.022028 \quad 3.24277934 \\
& \mathrm{H} \quad 0.667447647 \quad-1.343708 \quad 2.62854815 \\
& \text { O }-5.90071107-1.569495-1.28536908 \\
& 0-2.39817005-0.787501-1.58345186 \\
& \begin{array}{lllll}
0 & -2.45641842 & -1.778761 & 0.79940382
\end{array} \\
& \text { O }-0.180746881-2.5089892-0.765079136 \\
& \text { C }-4.89661546-0.473602-3.14612825 \\
& \text { C }-4.7420872-1.1025014-1.82282137 \\
& \text { C }-3.57782962-1.2236554-1.12277847 \\
& \text { C }-3.58154222-1.7754568 \quad 0.175236544 \\
& \text { C }-4.81022296-2.2600728 \quad 0.694623206 \\
& \text { C }-5.89627869-2.1400202-0.053293588 \\
& \text { C } 0.032677831 \quad 0.7500534 \quad-2.51834785 \\
& \text { H }-4.78159995-0.958218-3.71248554 \\
& \mathrm{H}-4.12440793 \quad 0.088112-3.40536994 \\
& \mathrm{H}-5.61133587 \quad 0.088112-3.2879434 \\
& \text { H }-4.89763819-2.577276 \quad 1.56267639 \\
& \mathrm{H}-6.79481052-2.390038 \quad 0.171623419 \\
& \text { H } \quad 0.704359343 \quad 1.189512 \quad-2.78210595 \\
& \text { H }-0.214483688-0.022028-3.24277934
\end{aligned}
$$


$\mathrm{H}-0.667447647 \quad 1.343708-2.62854815$

\section{References}

S1. Luo, S.; I. Rivalta, V. B.; Truhlar, D. G. Noncollinear Spins Provide a Self-Consistent Treatment of the Low-Spin State of a Biomimetic Oxomanganese Synthetic Trimer Inspired by the Oxygen Evolving Complex of Photosystem II. Phys. Chem. Lett. 2012, 2, 2629-2633.

S2. Sun, Y.; Melchior, M.; Summers, D. A.; Thompson, R. C.; Rettig, S. J.; Orvig, C. [( $\mu-$ $\left.\mathrm{OCH}_{3} \mathrm{VO}(\mathrm{ma})\right]_{2}$, A Strongly Antiferromagnetic Oxovanadium(IV) Dimer. Inorg. Chem. 1998, $37,3119$. 